\title{
Detection of Reactive Tetrahedral Intermediates in a Deep Cavitand with an Introverted Functionality
}

\section{Supporting Information}

\author{
Richard J. Hooley, Tetsuo Iwasawa and Julius Rebek, Jr.* \\ The Skaggs Institute for Chemical Biology and the Department of Chemistry, The Scripps \\ Research Institute MB-26, 10550 North Torrey Pines Rd., La Jolla, CA 92037. \\ jrebek@scripps.edu
}

\section{HRMS Characterization}

ESI-HRMS analysis for imine 15i (from 3 and isobutylamine): calc. for $\mathrm{C}_{134} \mathrm{H}_{168} \mathrm{~N}_{9} \mathrm{O}_{14}$ $\left(\mathrm{M}+\mathrm{H}^{+}\right):$2127.2705; found 2127.2715.

ESI-HRMS analysis for imine 16i (from 3 and $n$-propylamine): calc. for $\mathrm{C}_{133} \mathrm{H}_{166} \mathrm{~N}_{9} \mathrm{O}_{14}$ $\left(\mathrm{M}+\mathrm{H}^{+}\right): 2113.2548$; found 2113.2562.

ESI-HRMS analysis for imine 17i (from 3 and isopropylamine): calc. for $\mathrm{C}_{133} \mathrm{H}_{166} \mathrm{~N}_{9} \mathrm{O}_{14}$ $\left(\mathrm{M}+\mathrm{H}^{+}\right): 2113.2548$; found 2113.2518

ESI-HRMS analysis for imine 18i (from 3 and $n$-butylamine): calc. for $\mathrm{C}_{134} \mathrm{H}_{168} \mathrm{~N}_{9} \mathrm{O}_{14}$ $\left(\mathrm{M}+\mathrm{H}^{+}\right): 2127.2705$; found 2127.2717.

MALDI-TOF-MS analysis for imine 19i (from 3 and cyclopropylamine): calc. for $\mathrm{C}_{133} \mathrm{H}_{163} \mathrm{~N}_{9} \mathrm{O}_{14}\left(\mathrm{M}+\mathrm{H}^{+}\right)$: 2111.2392; found 2111.29.

MALDI-TOF-MS analysis for imine 20i (from 3 and cyclobutylamine): calc. for $\mathrm{C}_{133} \mathrm{H}_{163} \mathrm{~N}_{9} \mathrm{O}_{14}\left(\mathrm{M}+\mathrm{H}^{+}\right)$: 2125.2548; found 2125.24.

ESI-HRMS analysis for hemiaminal $\mathbf{2 4 h}$ (from $\mathbf{3}$ and ethanolamine): calc. for $\mathrm{C}_{134} \mathrm{H}_{168} \mathrm{~N}_{9} \mathrm{O}_{14}\left(\mathrm{M}+\mathrm{H}^{+}\right)$: 2133.2446; found 2133.2379

ESI-HRMS analysis for imine $23 \mathbf{i}$ (from 3 and ethylenediamine): calc. for $\mathrm{C}_{132} \mathrm{H}_{164} \mathrm{~N}_{10} \mathrm{O}_{14}$ $\left(\mathrm{M}+\mathrm{H}^{+}\right): 2114.2501$; found 2114.2449.

ESI-HRMS analysis for imine 24i (from 3 and ethanolamine): calc. for $\mathrm{C}_{132} \mathrm{H}_{163} \mathrm{~N}_{9} \mathrm{O}_{15}$ $\left(\mathrm{M}+\mathrm{H}^{+}\right):$2115.2341; found 2115.2320. 


\section{NMR Spectra:}

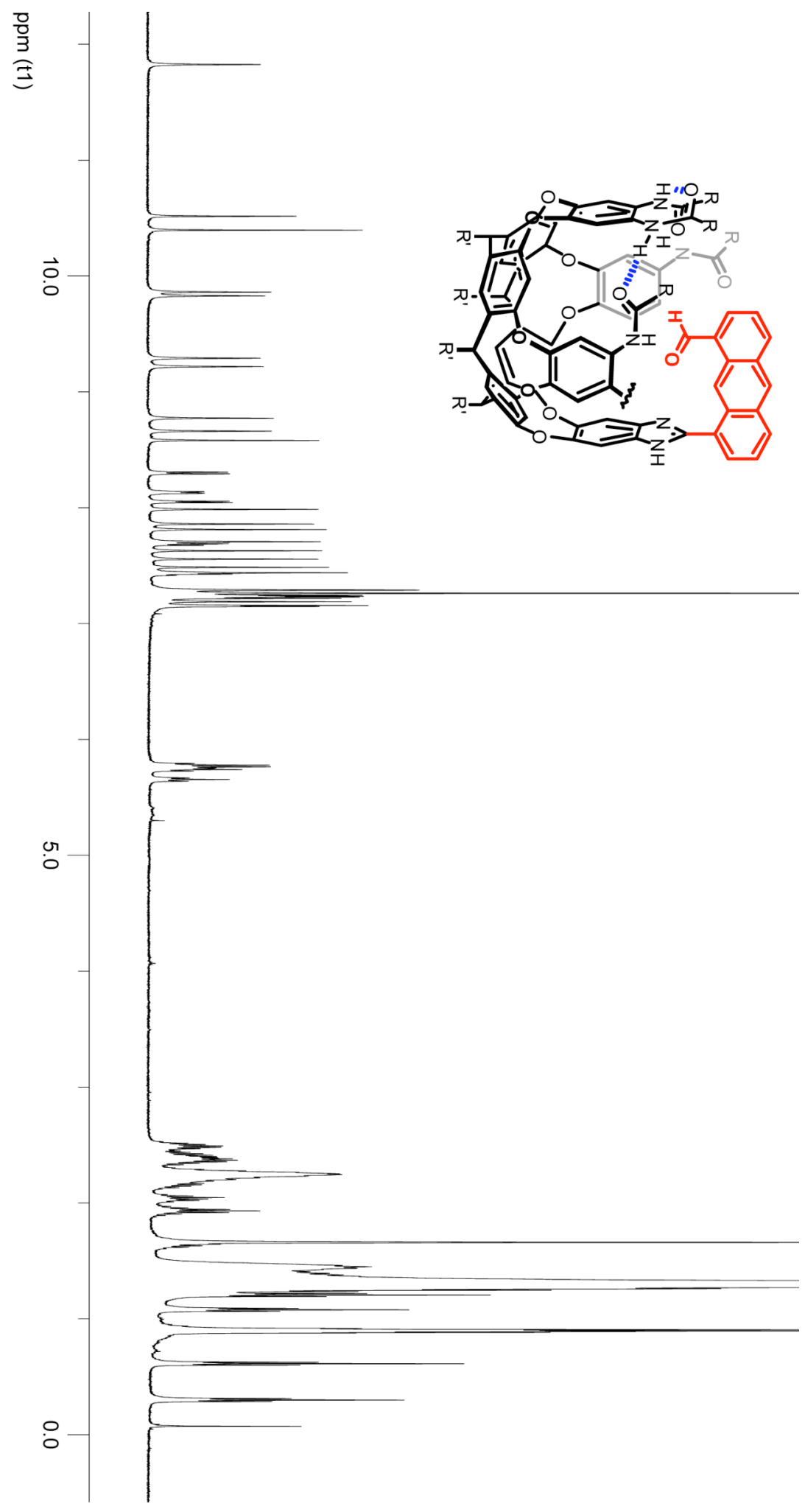

Figure $\boldsymbol{S}-\mathbf{1} .{ }^{1} \mathrm{H}$ NMR spectrum of cavitand $3\left(600 \mathrm{MHz}, \mathrm{CDCl}_{3}, 300 \mathrm{~K}\right)$ 


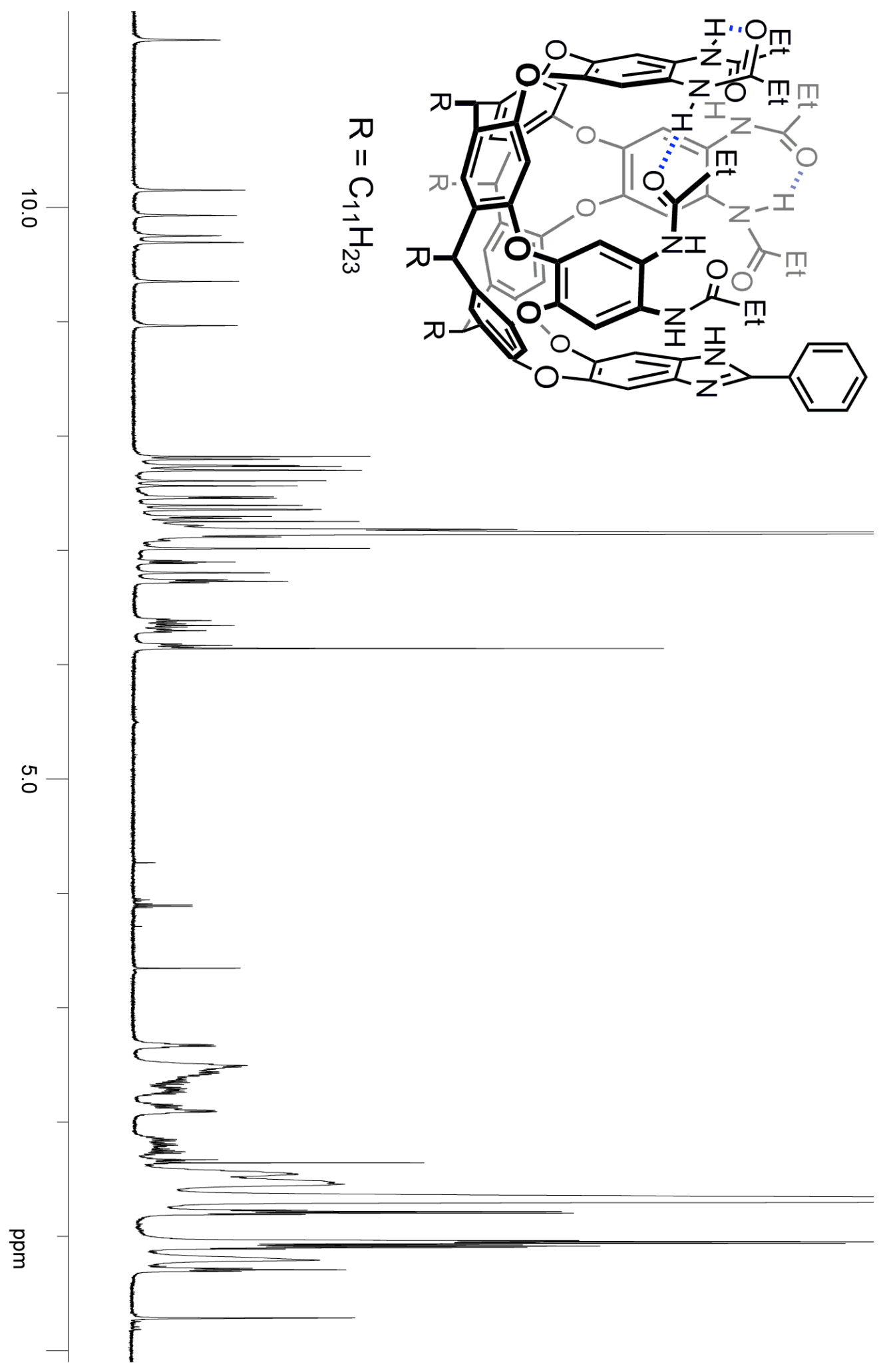

Figure $\boldsymbol{S}$-2. ${ }^{1} \mathrm{H}$ NMR spectrum of phenyl cavitand $7\left(600 \mathrm{MHz}, \mathrm{C}_{6} \mathrm{D}_{6}, 300 \mathrm{~K}\right)$ 


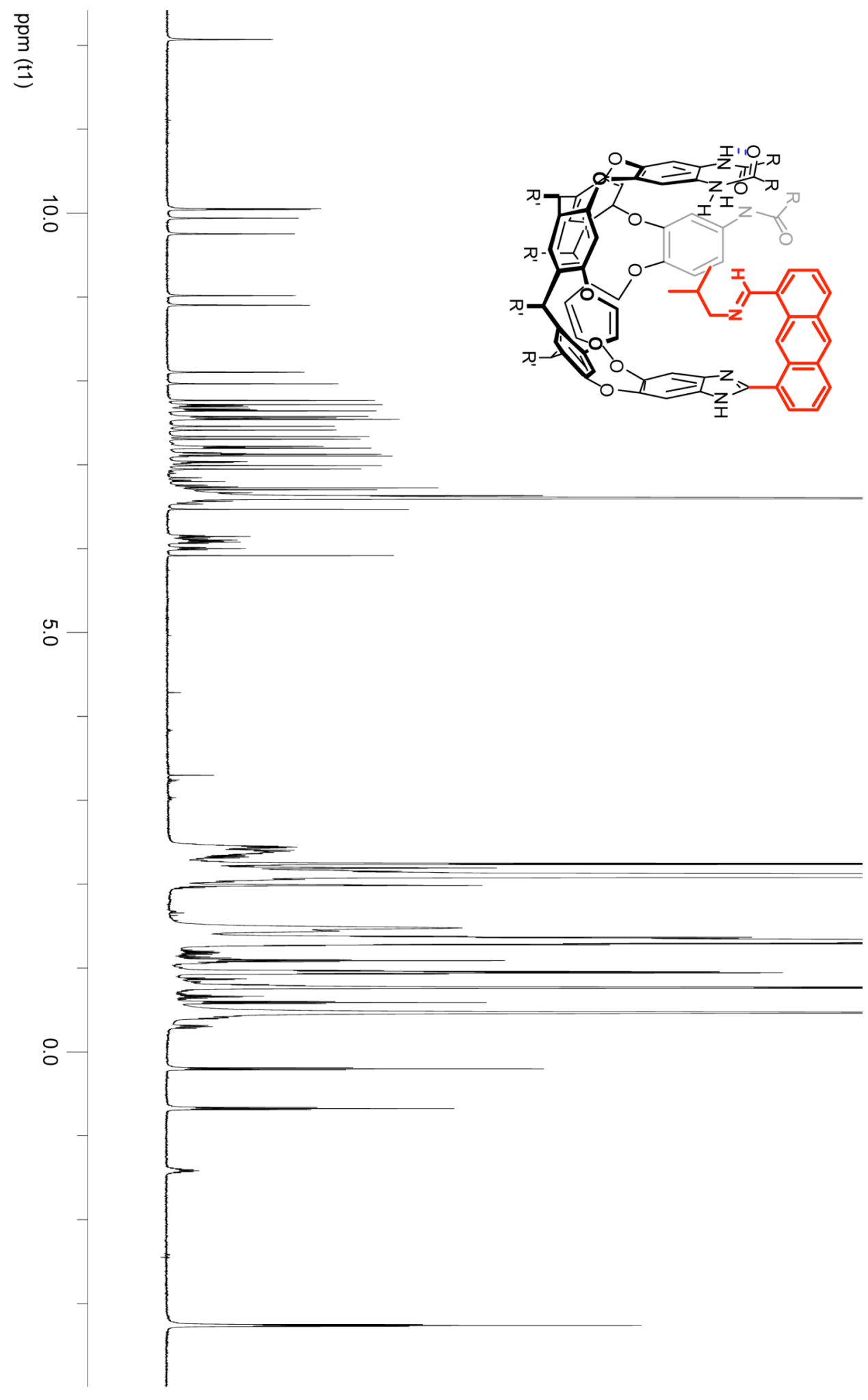

Figure $\boldsymbol{S}$-3. ${ }^{1}$ H NMR spectrum of imine 15i generated from exposure of cavitand $\mathbf{3}$ to isobutylamine (600 MHz, mesitylene- $\left.d_{12}, 300 \mathrm{~K}\right)$ 


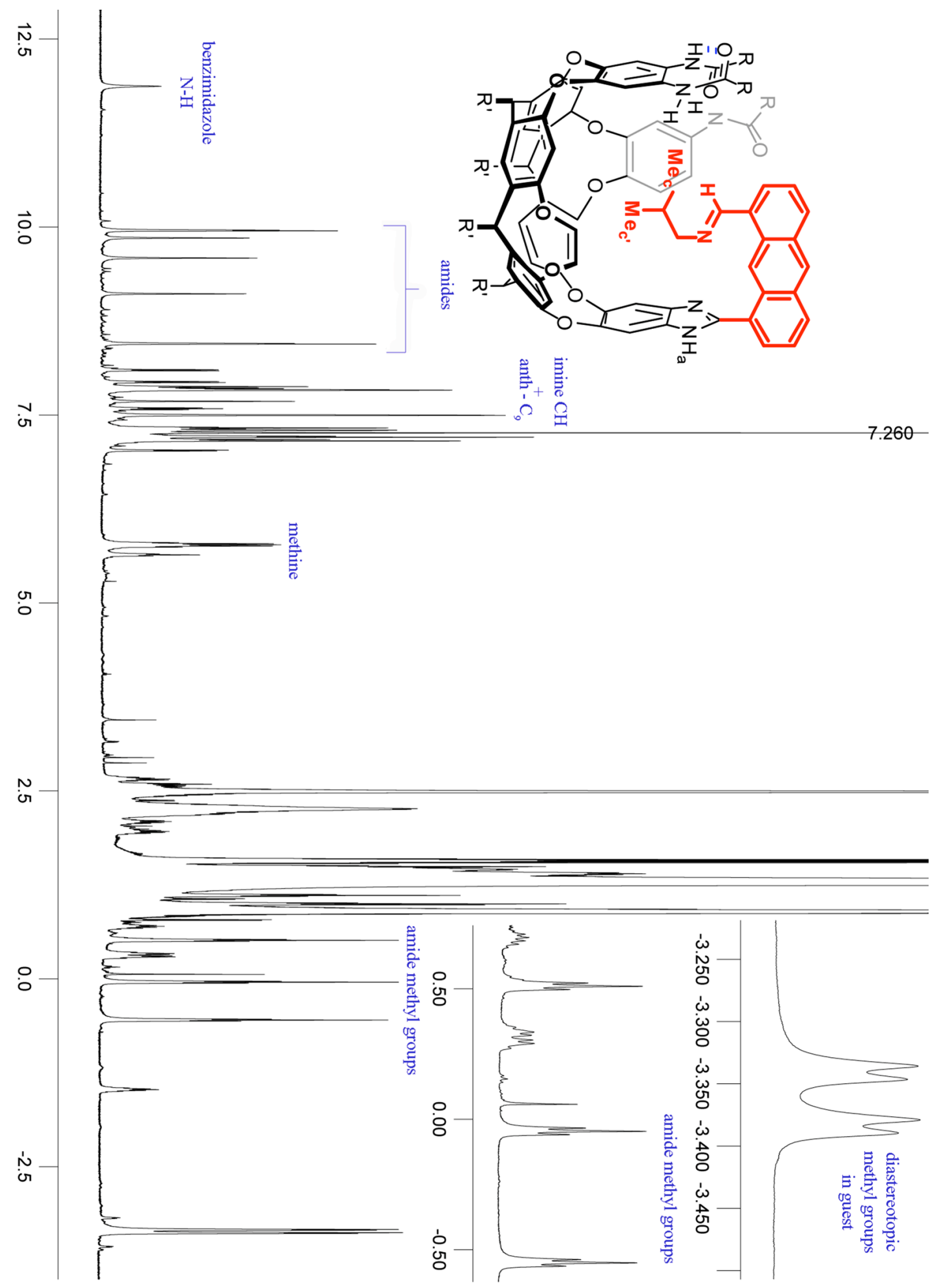

Figure $\boldsymbol{S}$-3b. Partially assigned ${ }^{1}$ H NMR spectrum of imine 15i generated from exposure of cavitand $\mathbf{3}$ to isobutylamine (600 MHz, mesitylene- $\left.d_{12}, 300 \mathrm{~K}\right)$. 


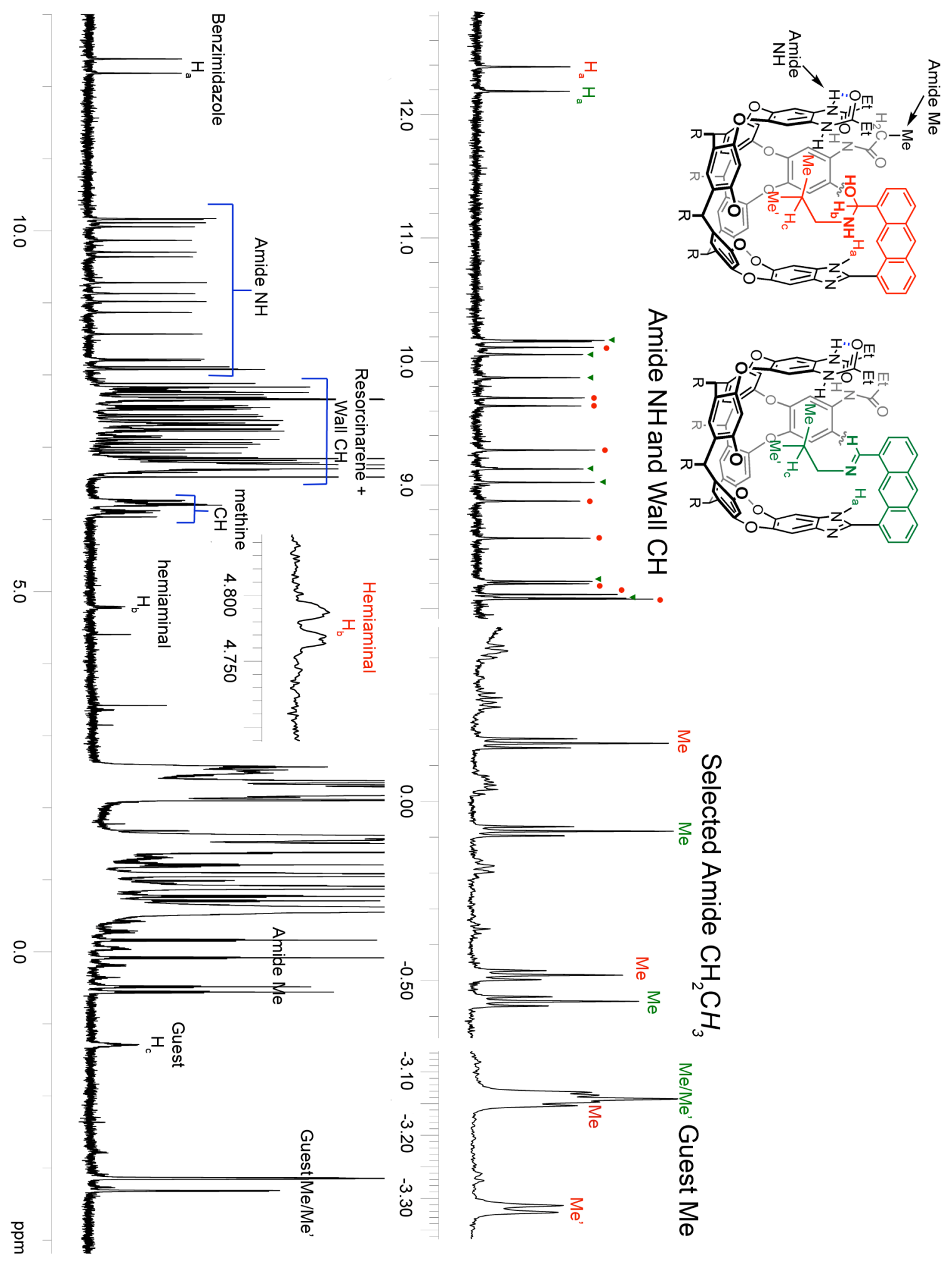

Figure $\boldsymbol{S}$-3c. Partially assigned ${ }^{1} \mathrm{H}$ NMR spectrum (with expansions) of hemiaminal/imine mixture $\mathbf{1 5}$ generated from exposure of cavitand 3 to isobutylamine for $30 \mathrm{~min}\left(600 \mathrm{MHz}\right.$, mesitylene- $\left.d_{12}, 300 \mathrm{~K}\right)$; Green annotation $=$ imine product peaks . Red annotation $=$ hemiaminal intermediate peaks. 


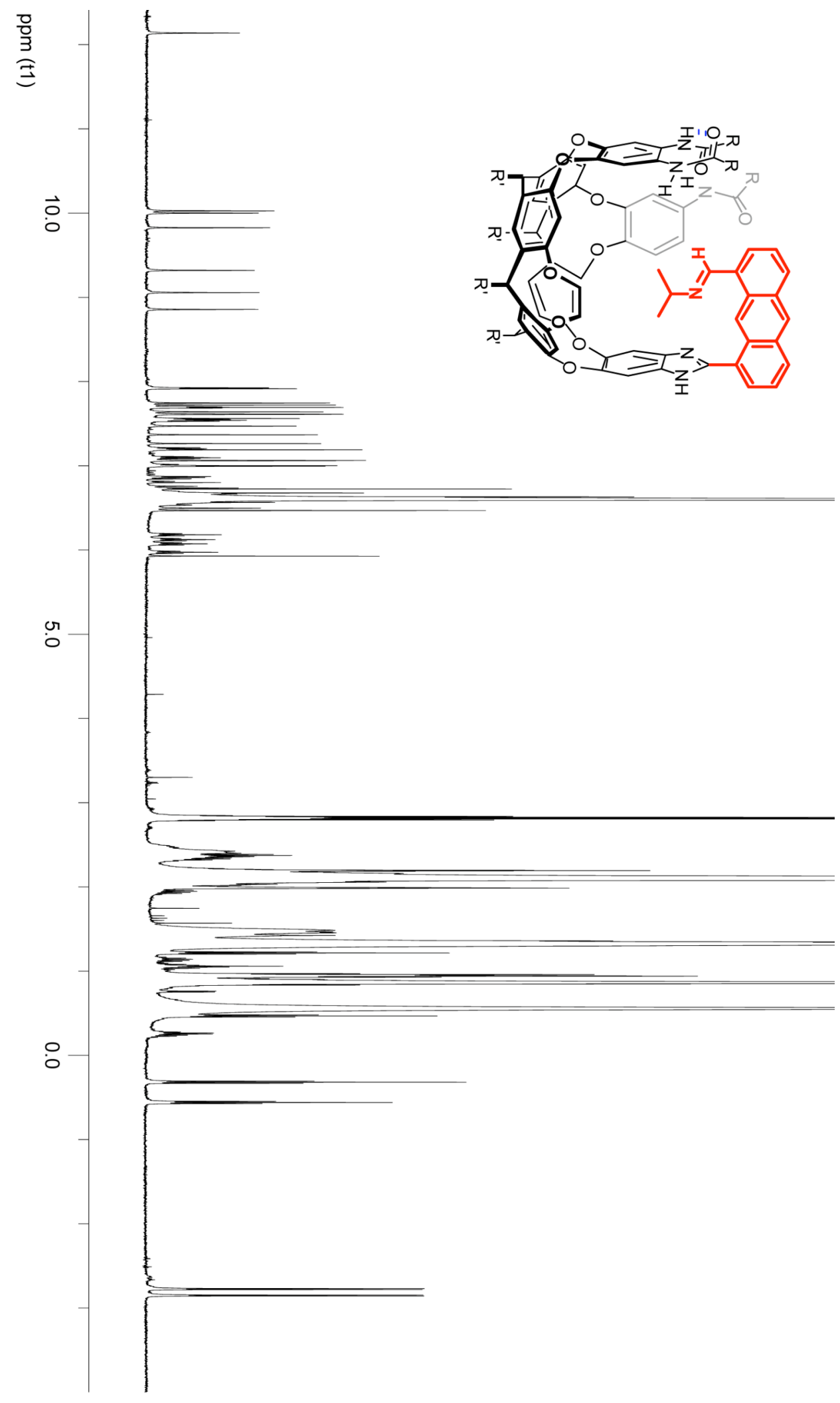

Figure $\boldsymbol{S}$-4. ${ }^{1} \mathrm{H}$ NMR spectrum of imine 17i generated from exposure of cavitand 3 to isopropylamine (600 MHz, mesitylene- $d_{12}, 300 \mathrm{~K}$ ) 


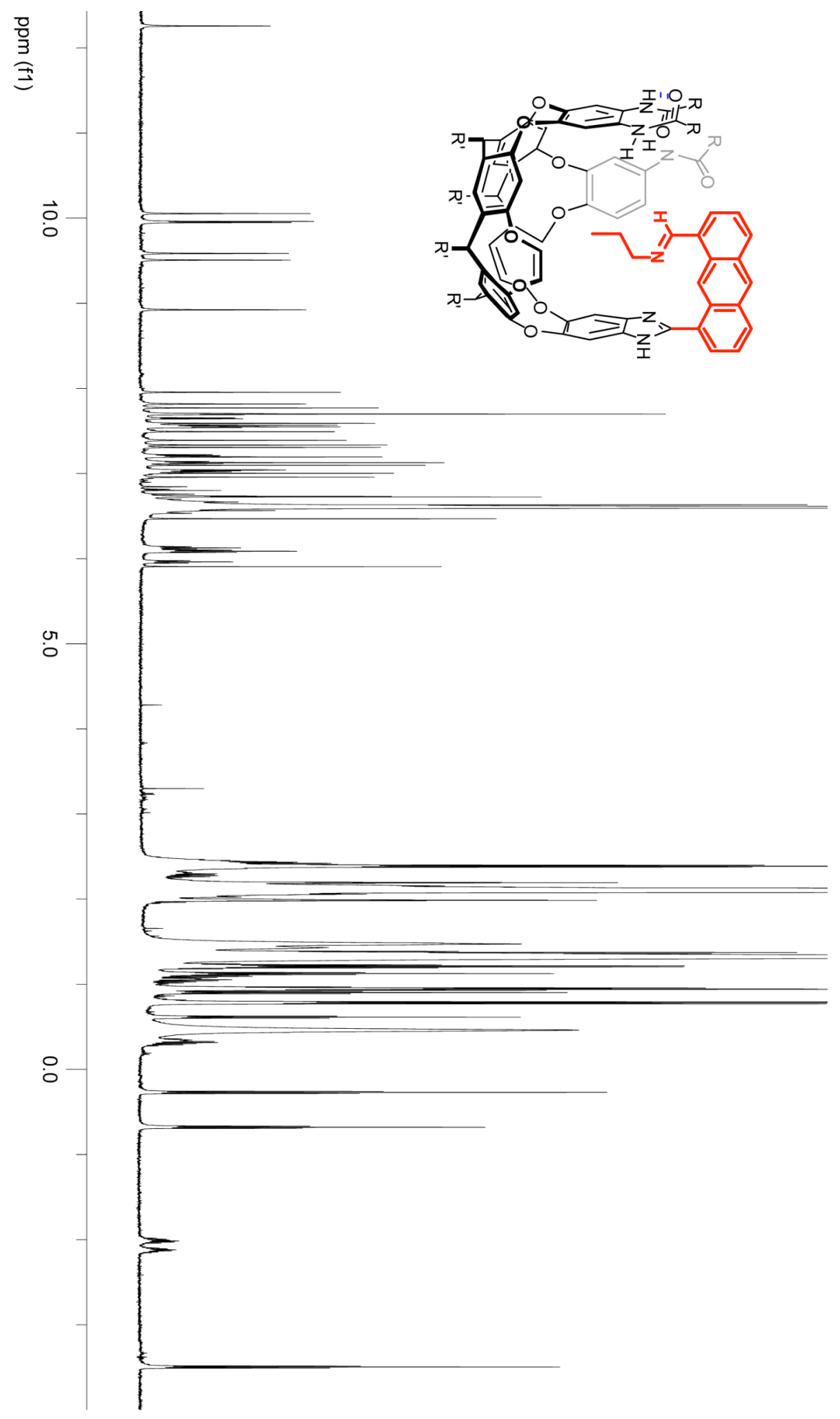

Figure $\boldsymbol{S}$-5. ${ }^{1} \mathrm{H}$ NMR spectrum of imine 16i generated from exposure of cavitand $\mathbf{3}$ to $n$-propylamine (600 MHz, mesitylene- $d_{12}, 300 \mathrm{~K}$ ) 


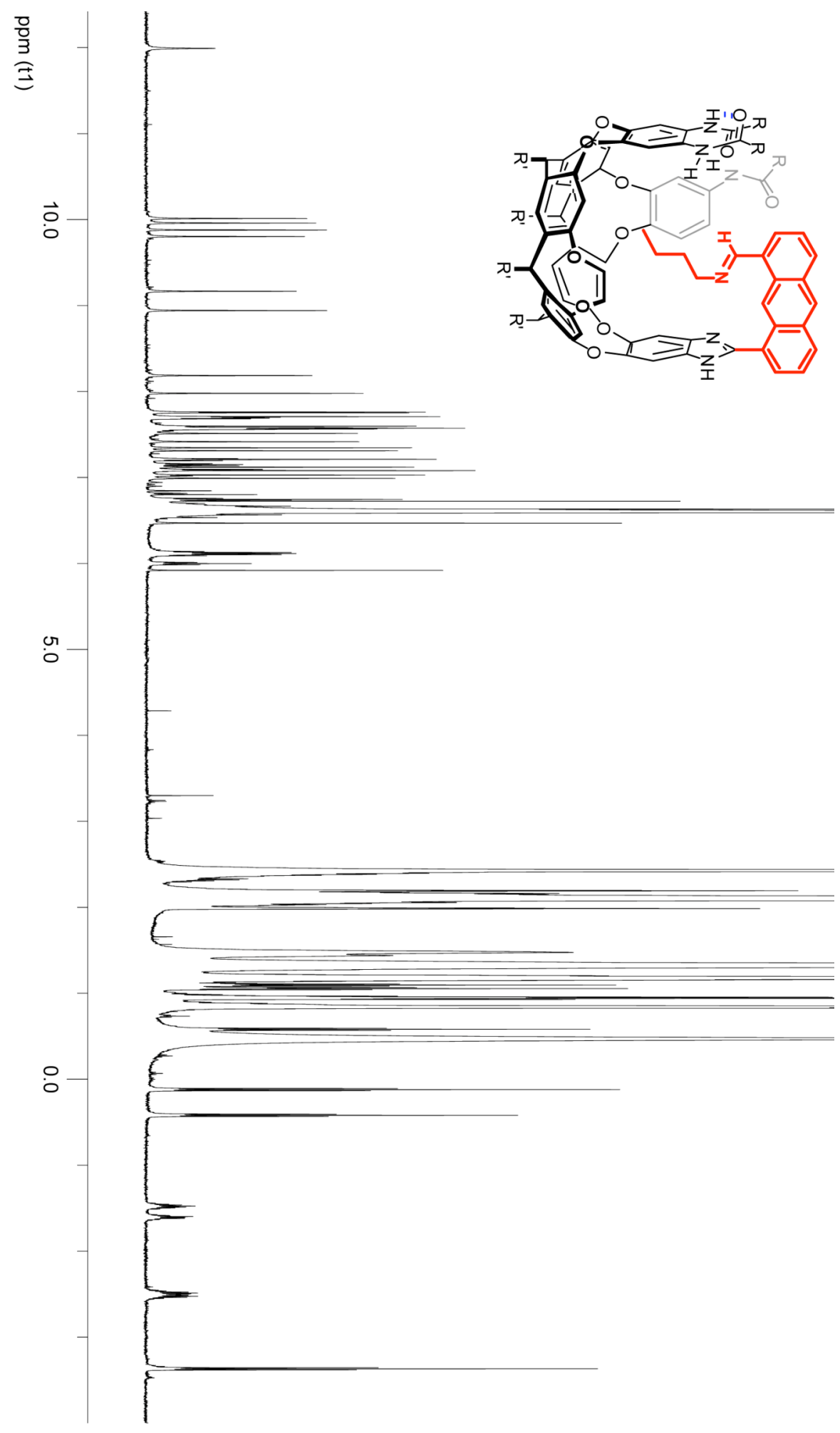

Figure $\boldsymbol{S}$-6. ${ }^{1} \mathrm{H}$ NMR spectrum of imine $\mathbf{1 8 i}$ generated from exposure of cavitand $\mathbf{3}$ to $n$-butylamine (600 MHz, mesitylene- $d_{12}, 300 \mathrm{~K}$ ) 


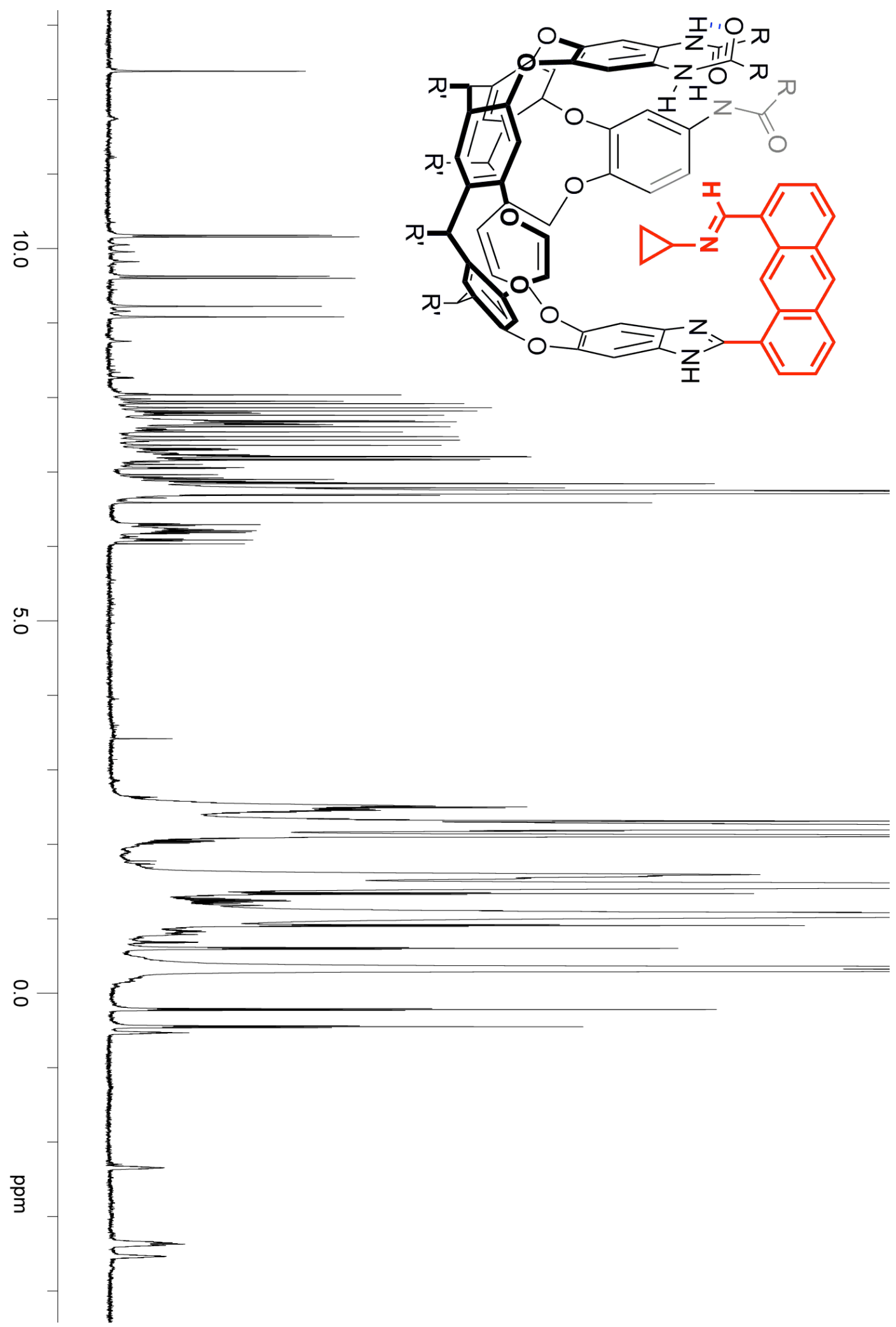

Figure $S$-7. ${ }^{1} \mathrm{H}$ NMR spectrum of imine 19i generated from exposure of cavitand $\mathbf{3}$ to cyclopropylamine (600 MHz, mesitylene- $\left.d_{12}, 300 \mathrm{~K}\right)$ 


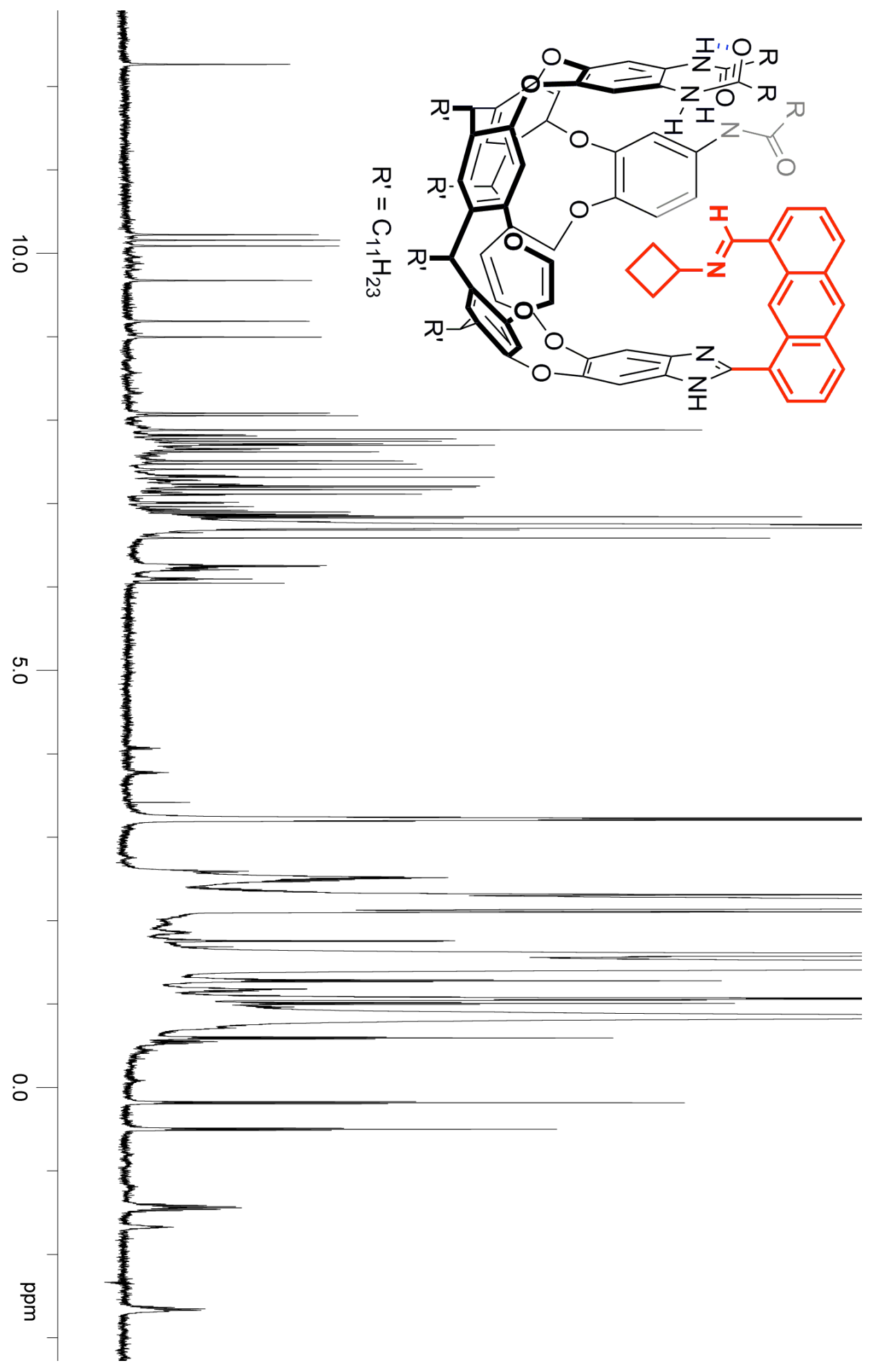

Figure S-8. ${ }^{1} \mathrm{H}$ NMR spectrum of imine 20i generated from exposure of cavitand $\mathbf{3}$ to cyclobutylamine (600 MHz, mesitylene- $\left.d_{12}, 300 \mathrm{~K}\right)$ 


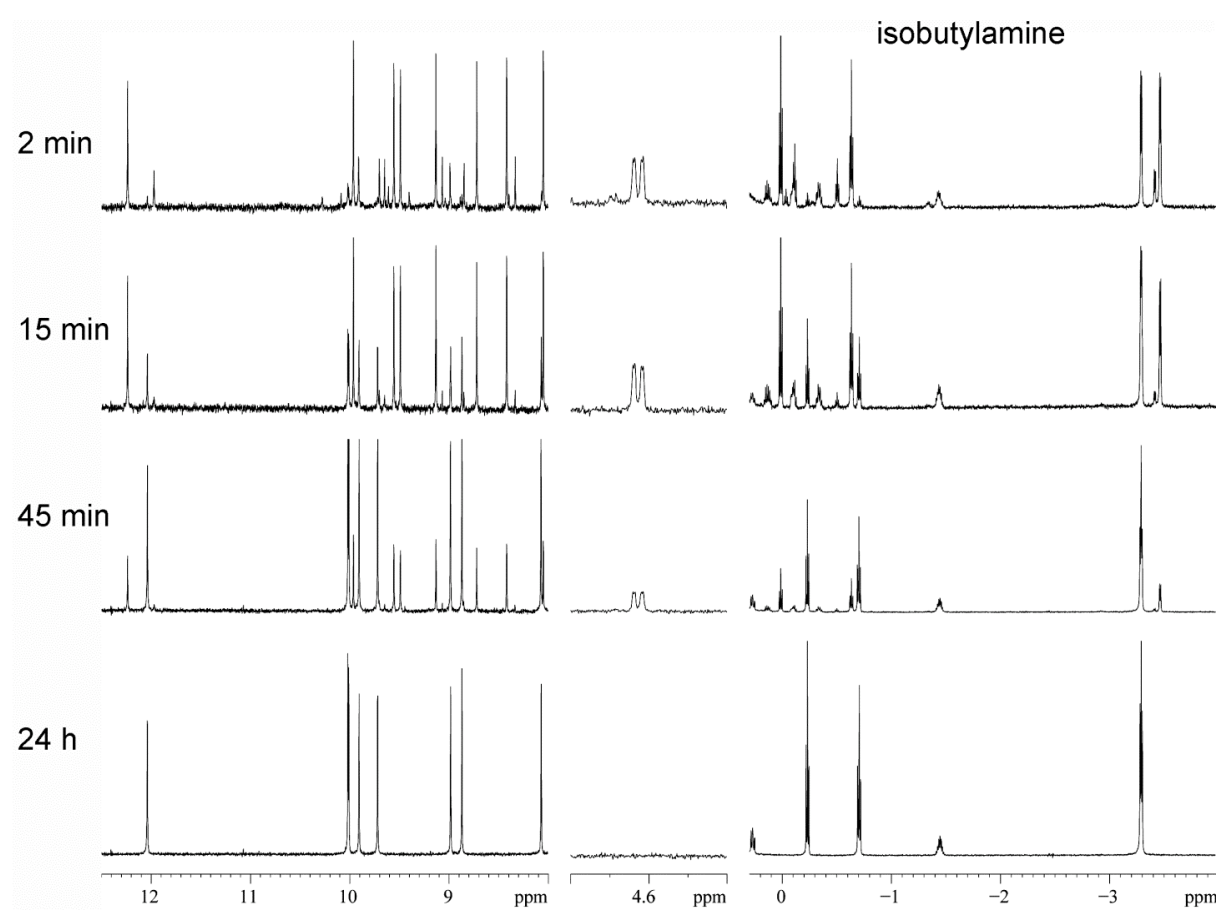

Figure $\boldsymbol{S}$-9. Sections of ${ }^{1} \mathrm{H}$ NMR spectra of the reaction of cavitand $\mathbf{3}$ with isobutylamine over time (600 MHz, mesitylene- $d_{12}, 300 \mathrm{~K}$ ).

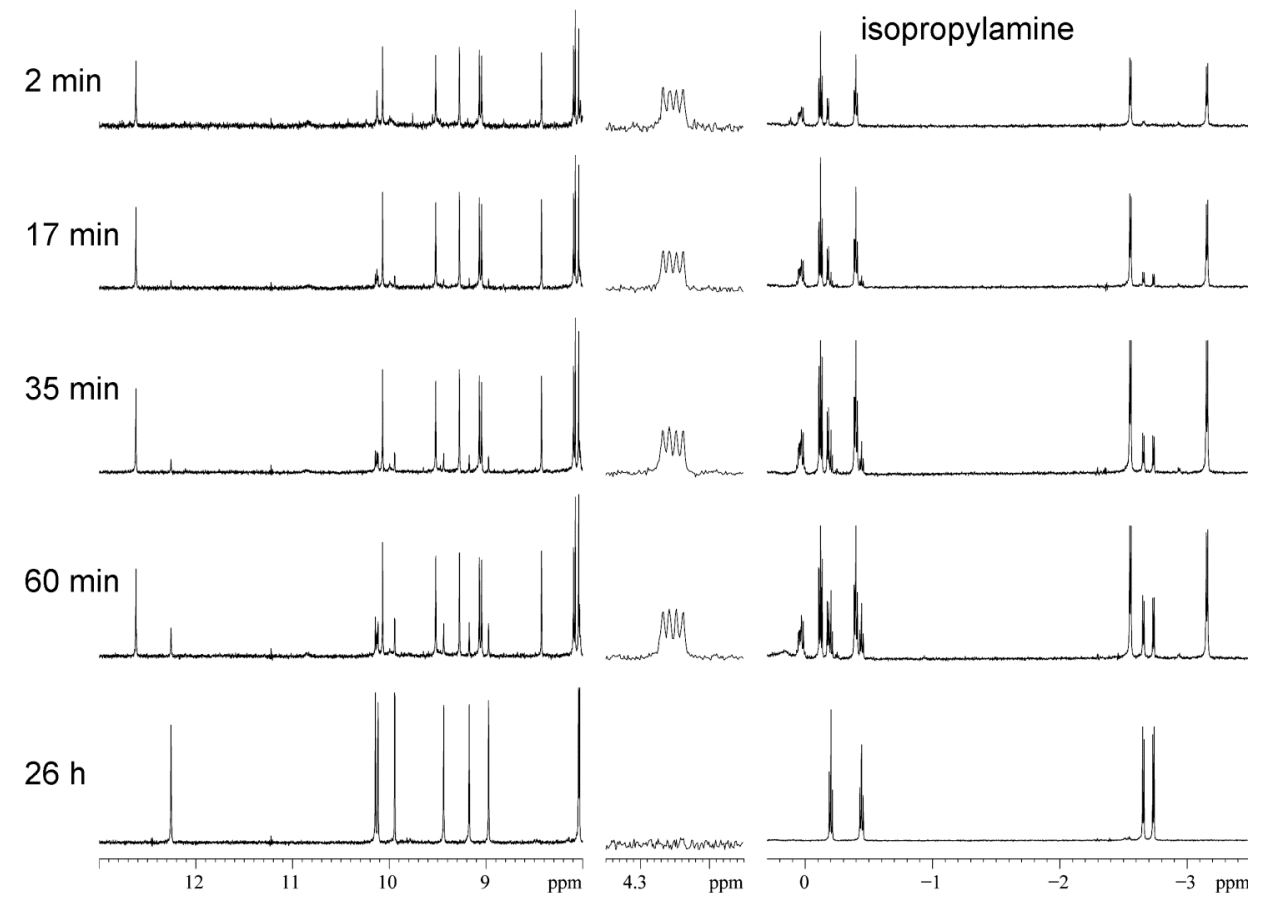

Figure $\boldsymbol{S}$-10. Sections of ${ }^{1}$ H NMR spectra of the reaction of cavitand 3 with isopropylamine over time (600 MHz, mesitylene- $d_{12}, 300 \mathrm{~K}$ ). 


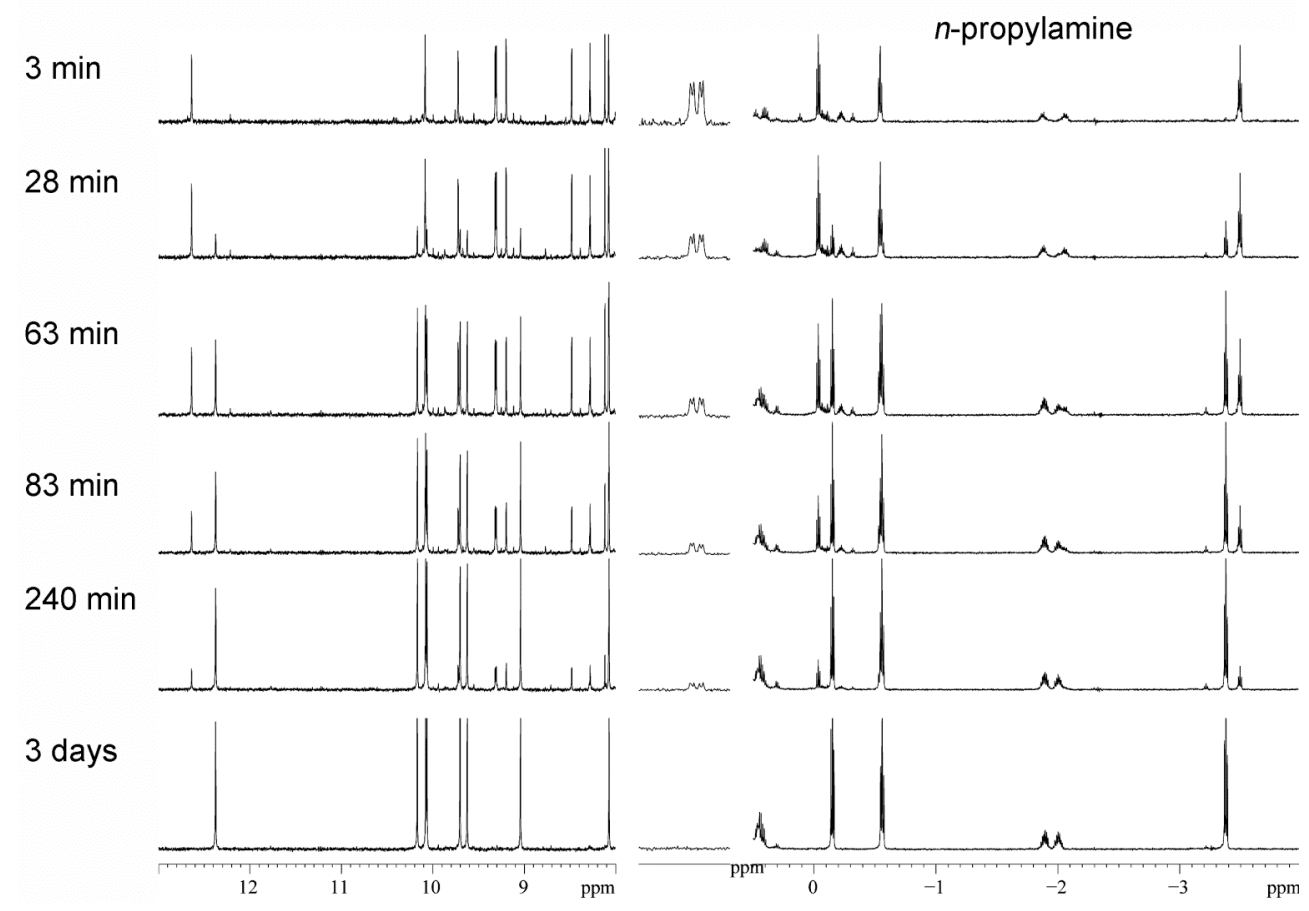

Figure $\boldsymbol{S}$-11. Sections of ${ }^{1} \mathrm{H}$ NMR spectra of the reaction of cavitand $\mathbf{3}$ with $n$-propylamine over time (600 MHz, mesitylene- $\left.d_{12}, 300 \mathrm{~K}\right)$.

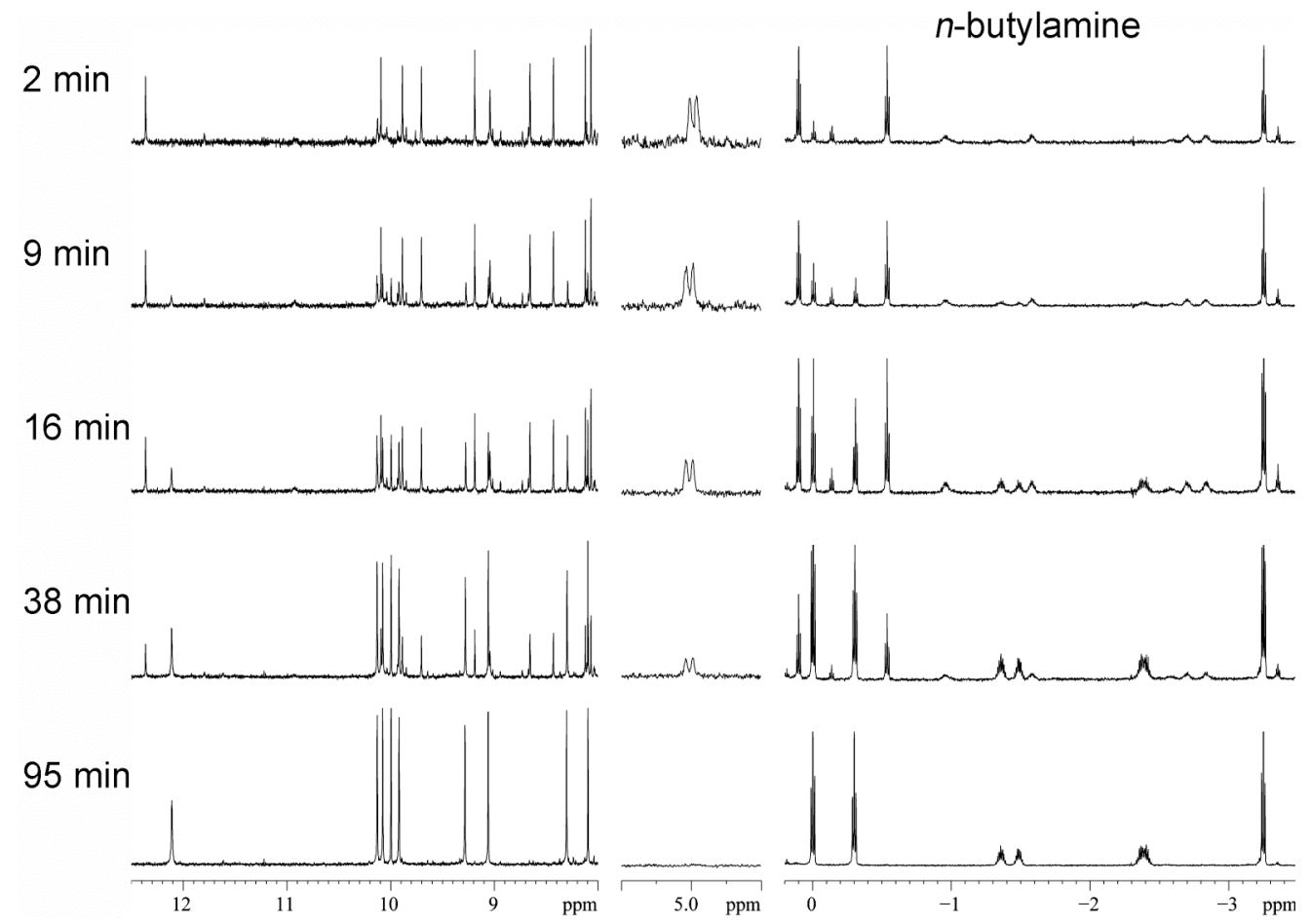

Figure $\boldsymbol{S}$-12. Sections of ${ }^{1} \mathrm{H}$ NMR spectra of the reaction of cavitand 3 with $n$-butylamine over time (600 MHz, mesitylene- $d_{12}, 300 \mathrm{~K}$ ). 


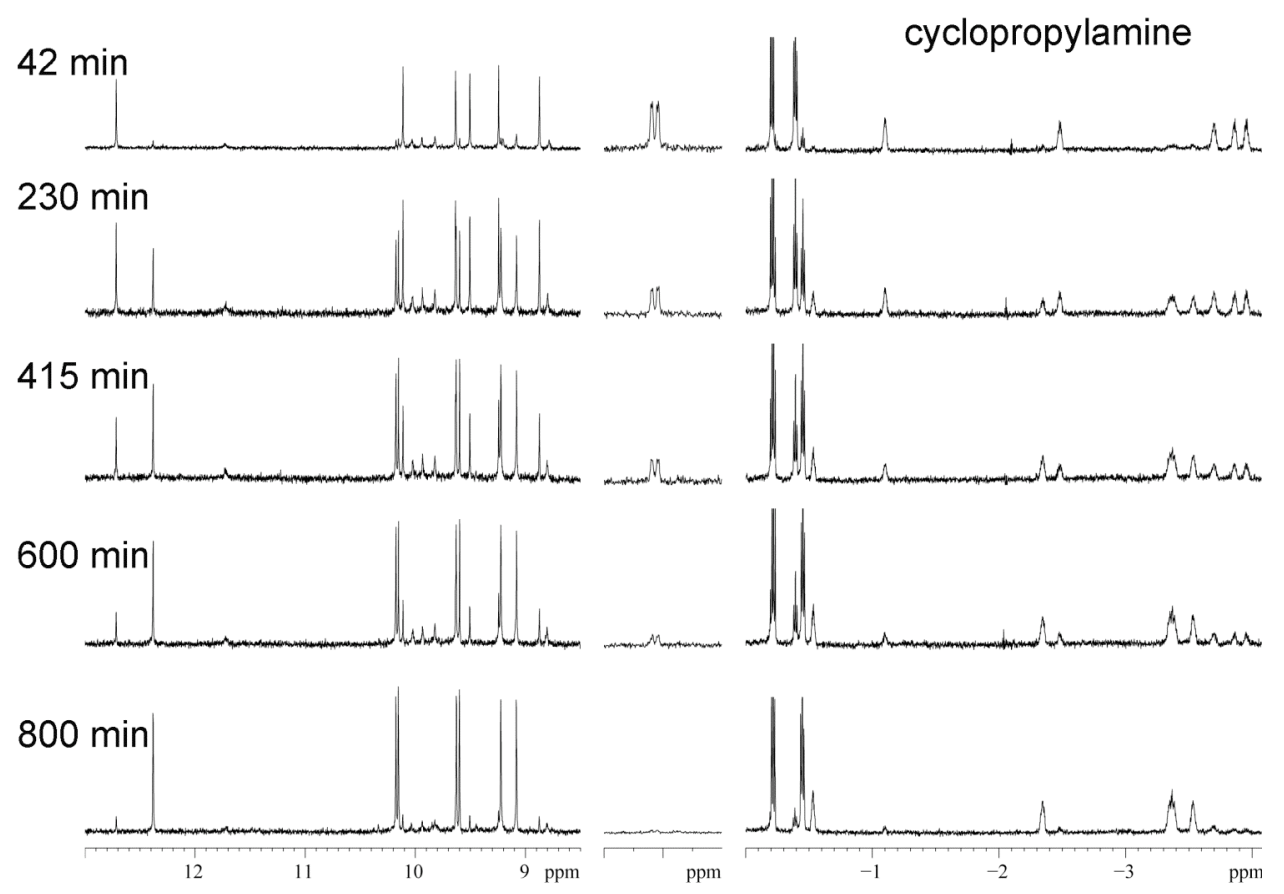

Figure $\boldsymbol{S}$-13. Sections of ${ }^{1} \mathrm{H}$ NMR spectra of the reaction of cavitand $\mathbf{3}$ with cyclopropylamine over time (600 MHz, mesitylene- $\left.d_{12}, 300 \mathrm{~K}\right)$. cyclobutylamine

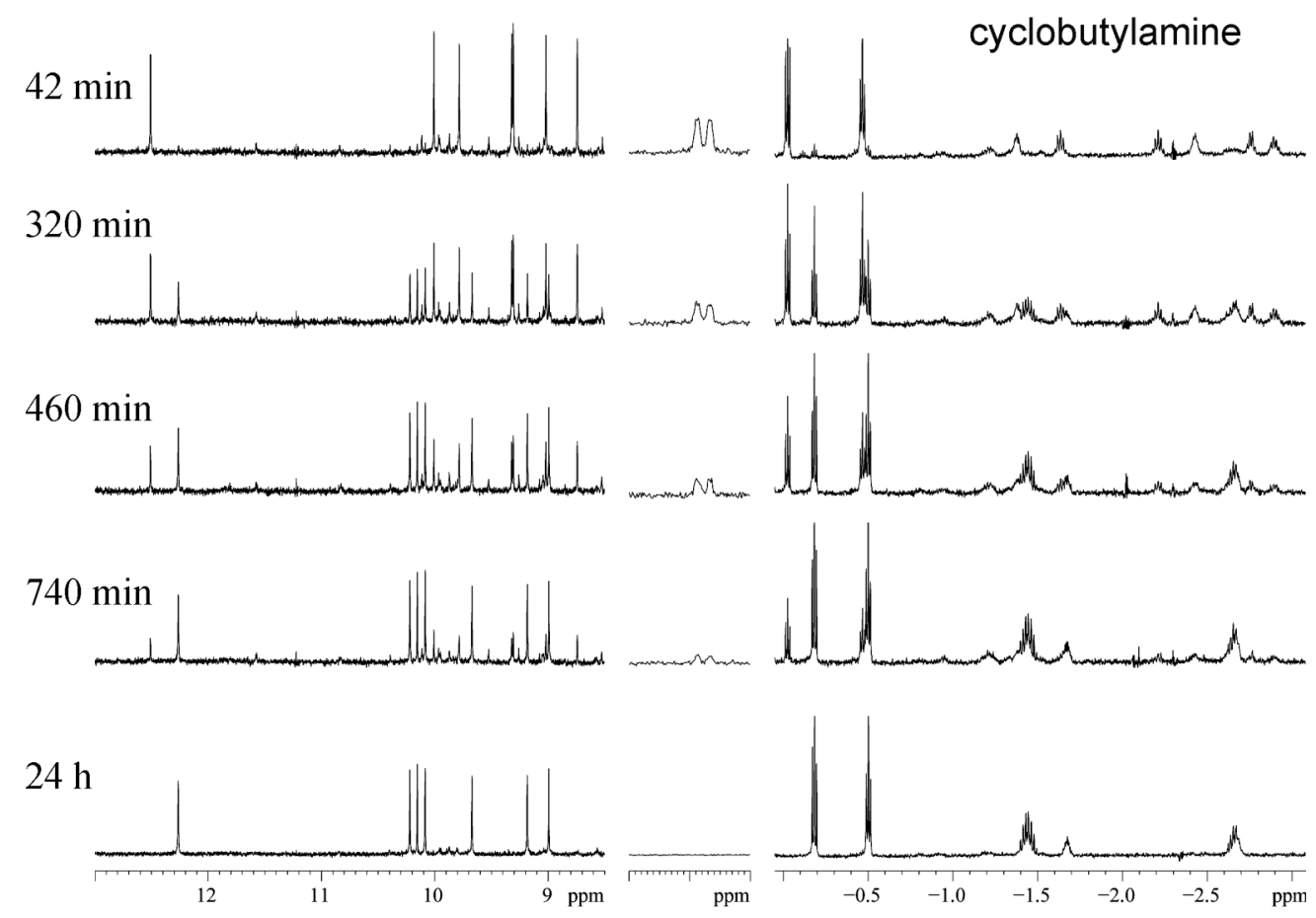

Figure $\boldsymbol{S}$-14. Sections of ${ }^{1} \mathrm{H}$ NMR spectra of the reaction of cavitand 3 with cyclobutylamine over time (600 MHz, mesitylene- $\left.d_{12}, 300 \mathrm{~K}\right)$. 

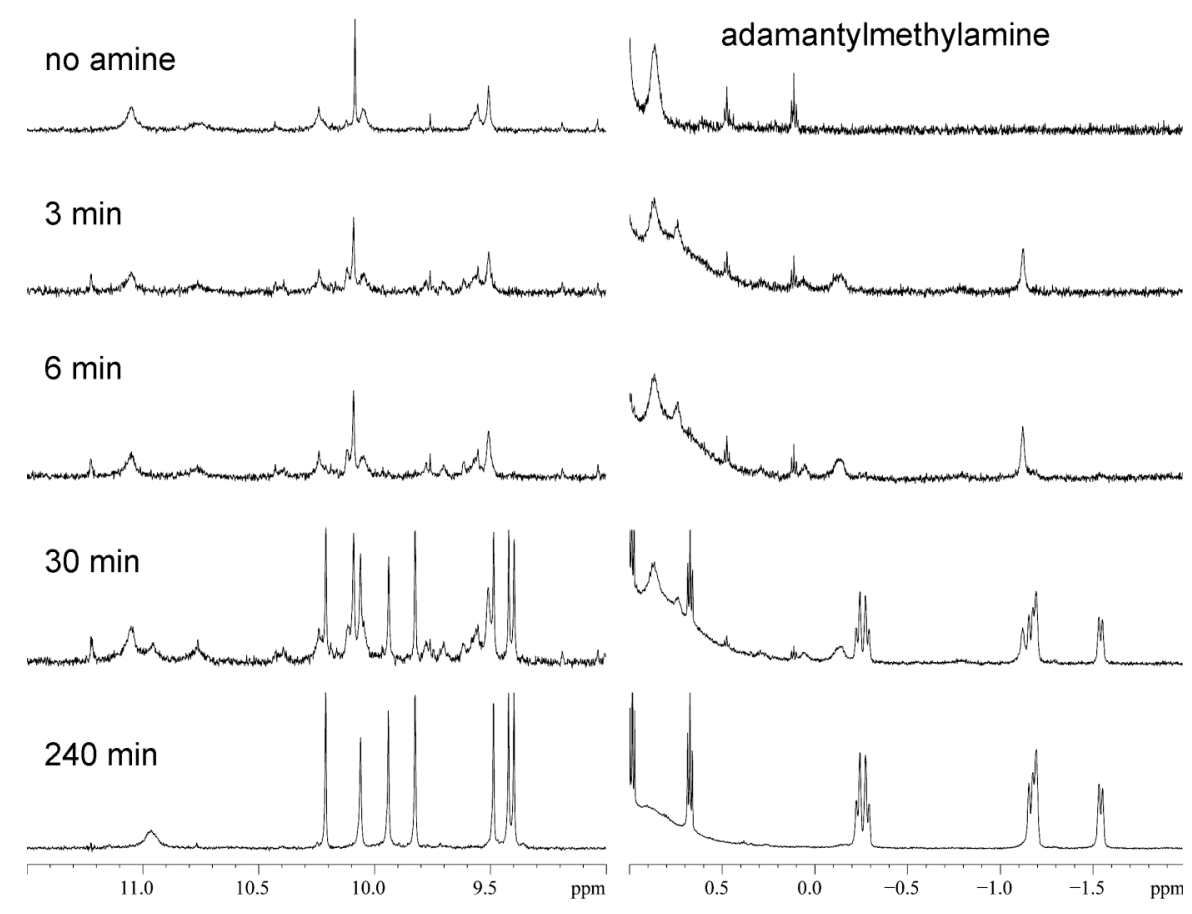

Figure $\boldsymbol{S}$-15. Sections of ${ }^{1} \mathrm{H}$ NMR spectra of the reaction of cavitand $\mathbf{3}$ with adamantylmethylamine over time $\left(600 \mathrm{MHz}\right.$, mesitylene- $\left.d_{12}, 300 \mathrm{~K}\right)$.

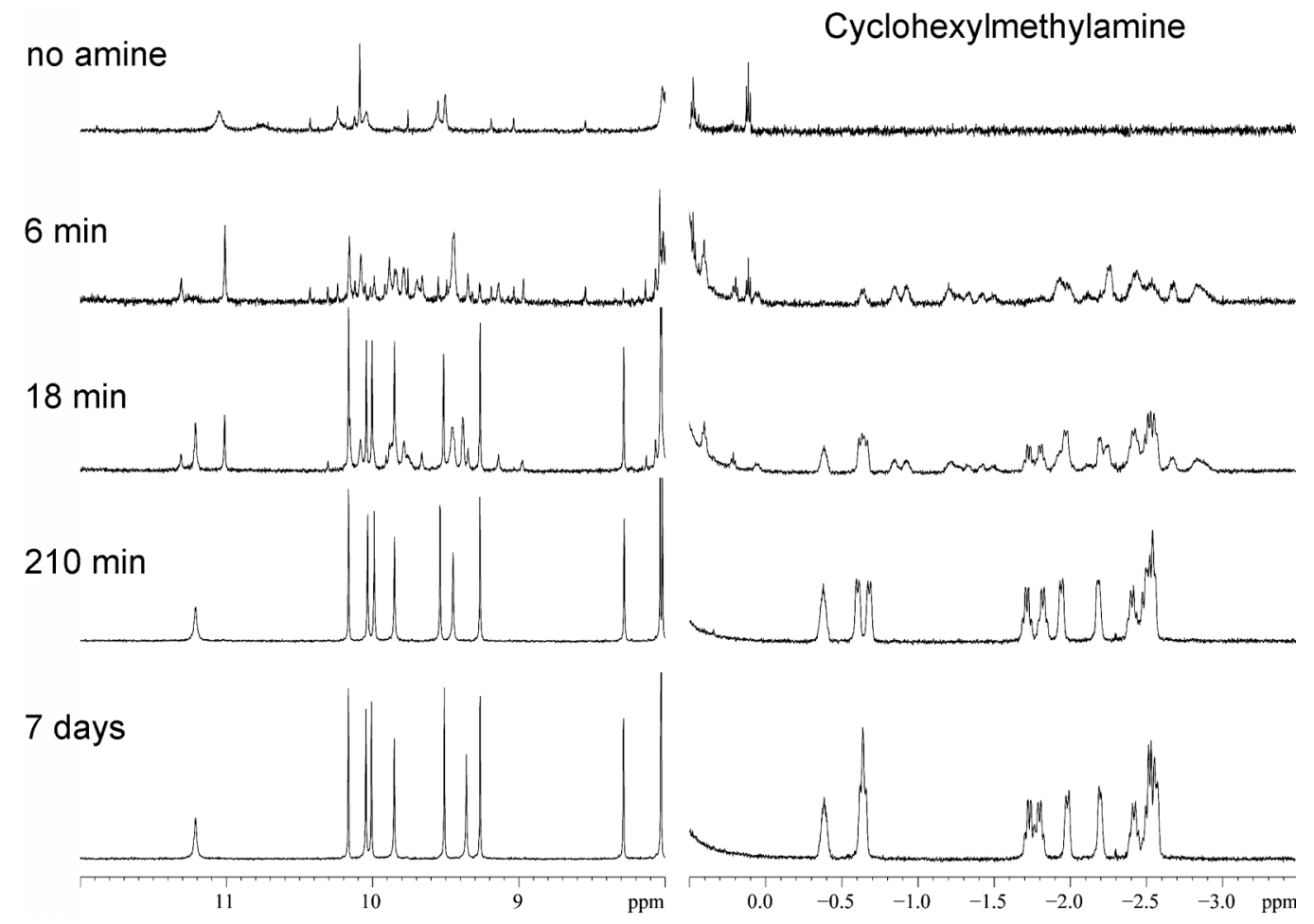

Figure $\boldsymbol{S}$-16. Sections of ${ }^{1} \mathrm{H}$ NMR spectra of the reaction of cavitand 3 with cyclohexylmethylamine over time (600 MHz, mesitylene- $\left.d_{12}, 300 \mathrm{~K}\right)$. 


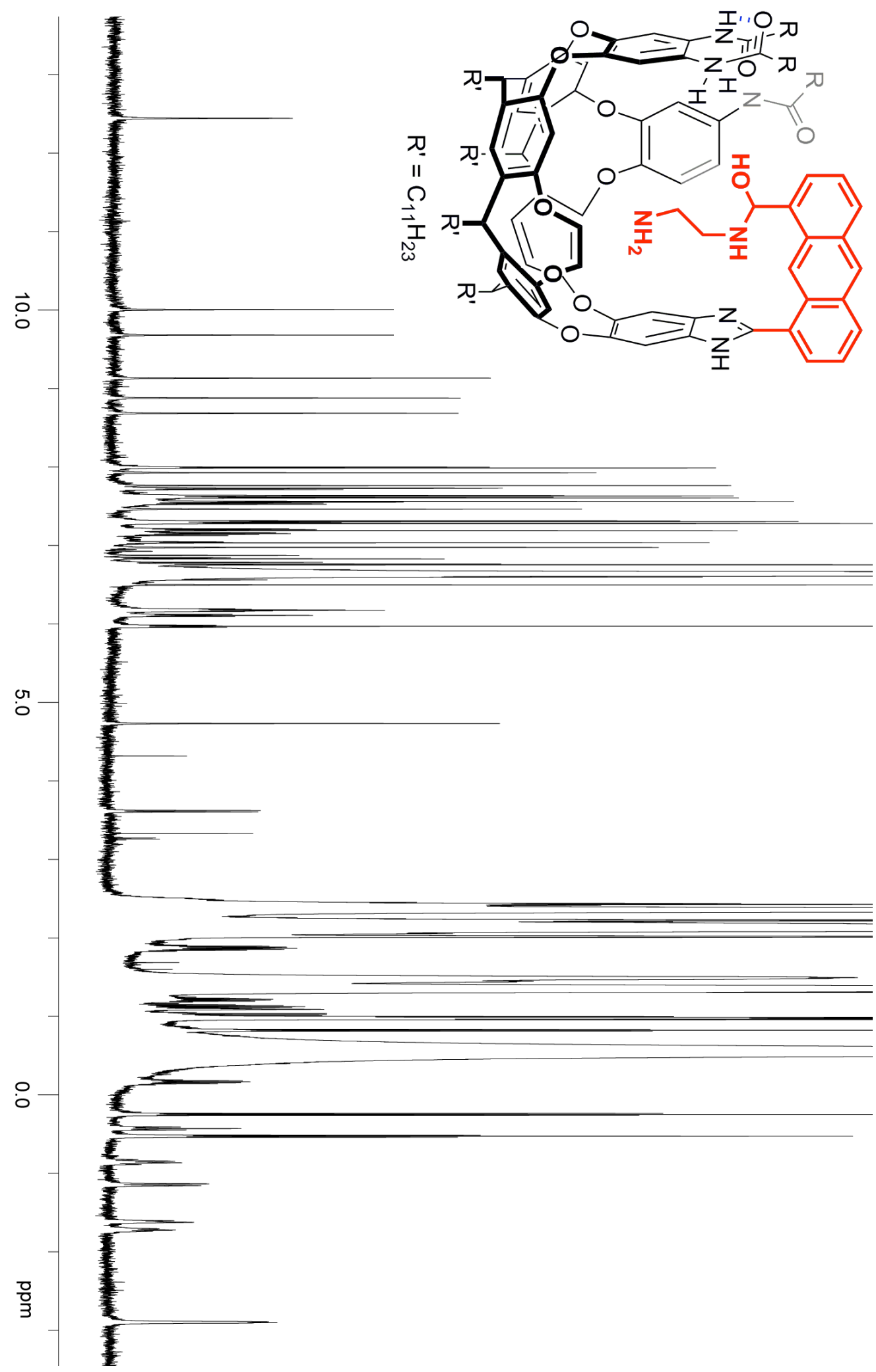

Figure $\boldsymbol{S - 1 7} .{ }^{1} \mathrm{H}$ NMR spectrum of hemiaminal $\mathbf{2 3 h}$ generated from exposure of cavitand $\mathbf{3}$ to ethylenediamine (spectrum taken after $10 \mathrm{~min}, 600 \mathrm{MHz}$, mesitylene- $d_{12}, 300 \mathrm{~K}$ ) 

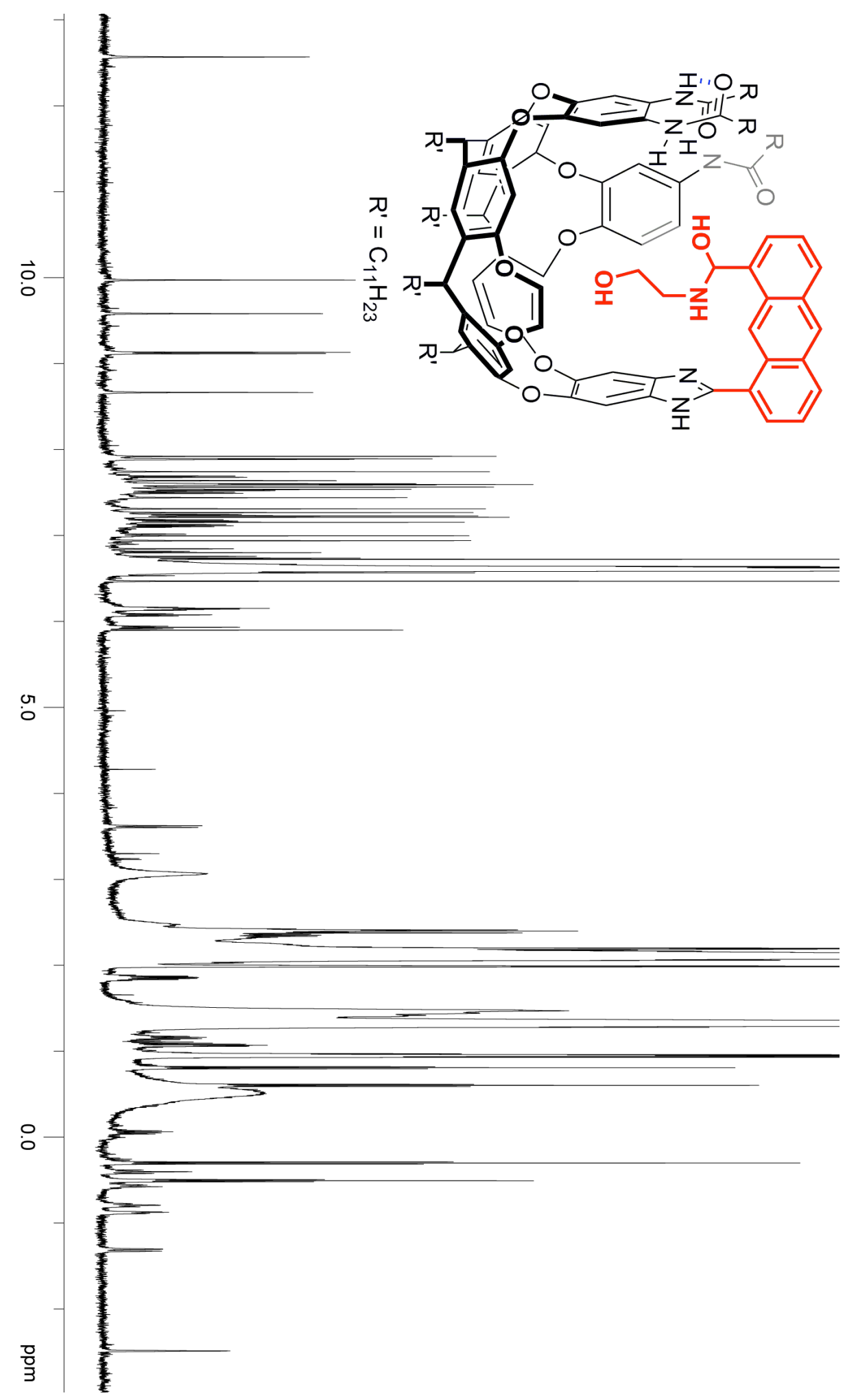

Figure $\boldsymbol{S}$-18. ${ }^{1} \mathrm{H}$ NMR spectrum of hemiaminal 24h generated from exposure of cavitand $\mathbf{3}$ to ethanolamine (spectrum taken after $10 \mathrm{~min}, 600 \mathrm{MHz}$, mesitylene- $d_{12}, 300 \mathrm{~K}$ ) 


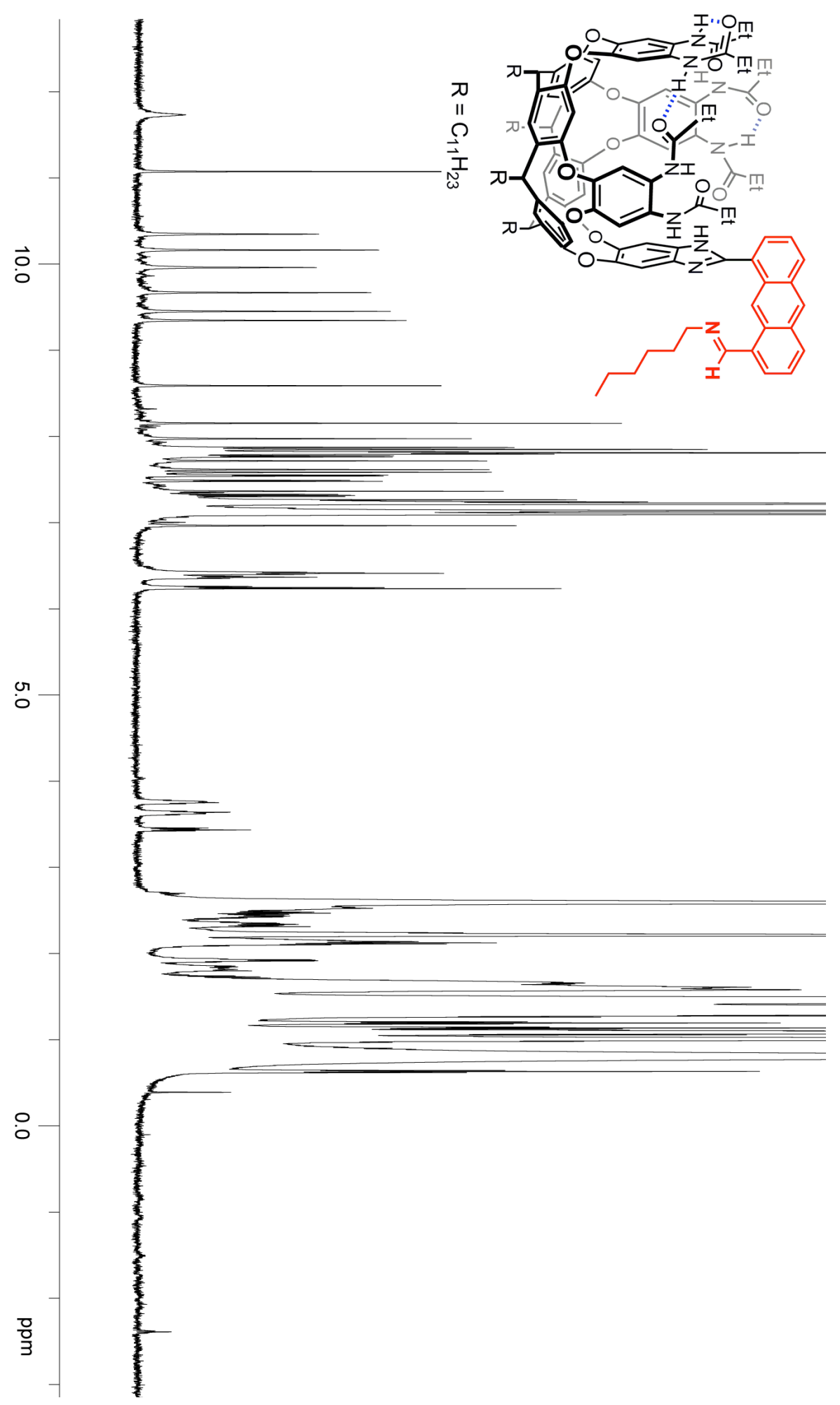

Figure $\boldsymbol{S}$-19. ${ }^{1} \mathrm{H}$ NMR spectrum of external imine generated from exposure of cavitand $\mathbf{3}$ to $n$-hexylamine (600 MHz, toluene- $\left.d_{8}, 300 \mathrm{~K}\right)$ 


\section{Selected NMR Experiments}

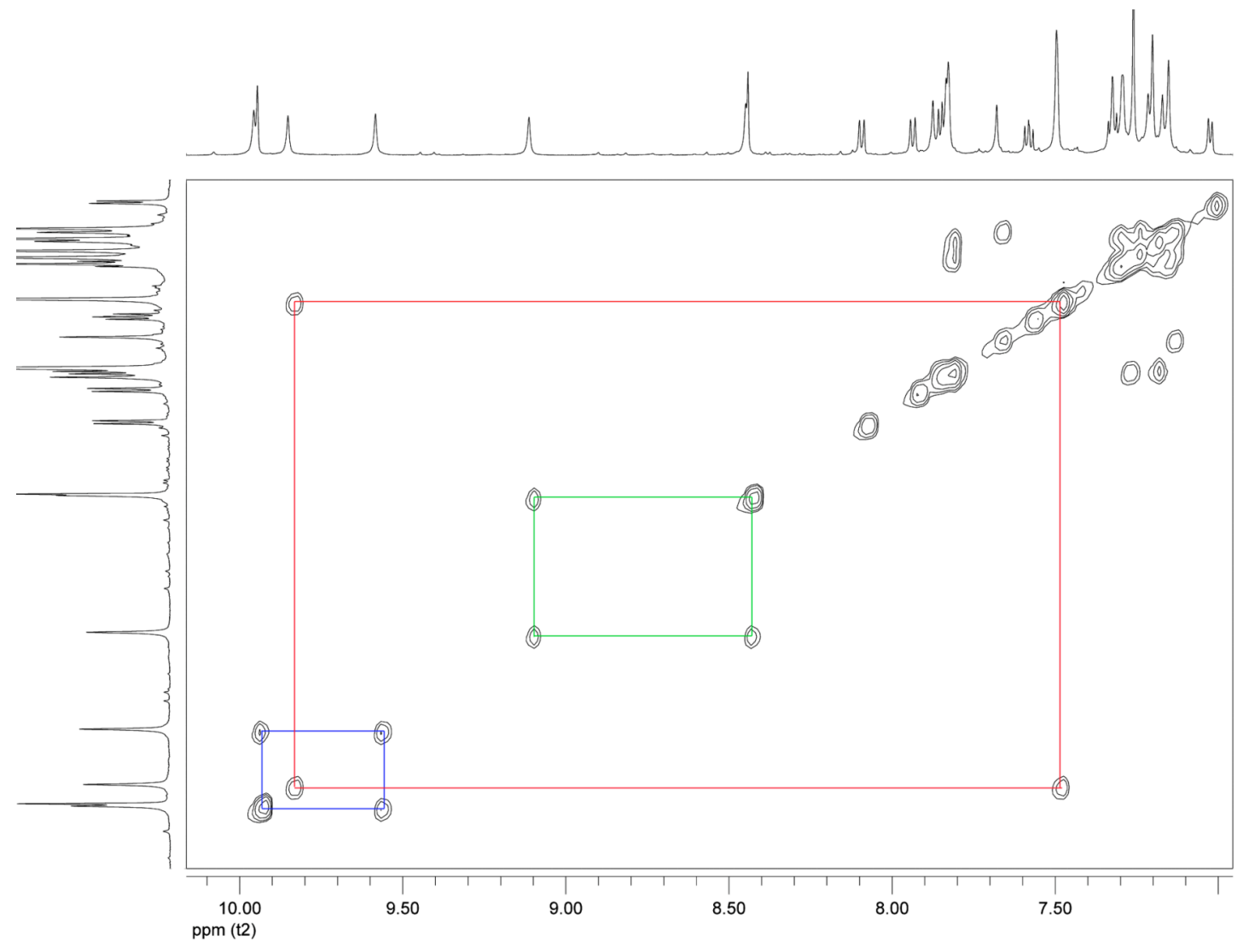

Figure $\boldsymbol{S}$-20. Downfield region of the 2D NOESY spectrum of isobutyl imine cavitand 15i, showing chemical exchange between amide NH peaks. 


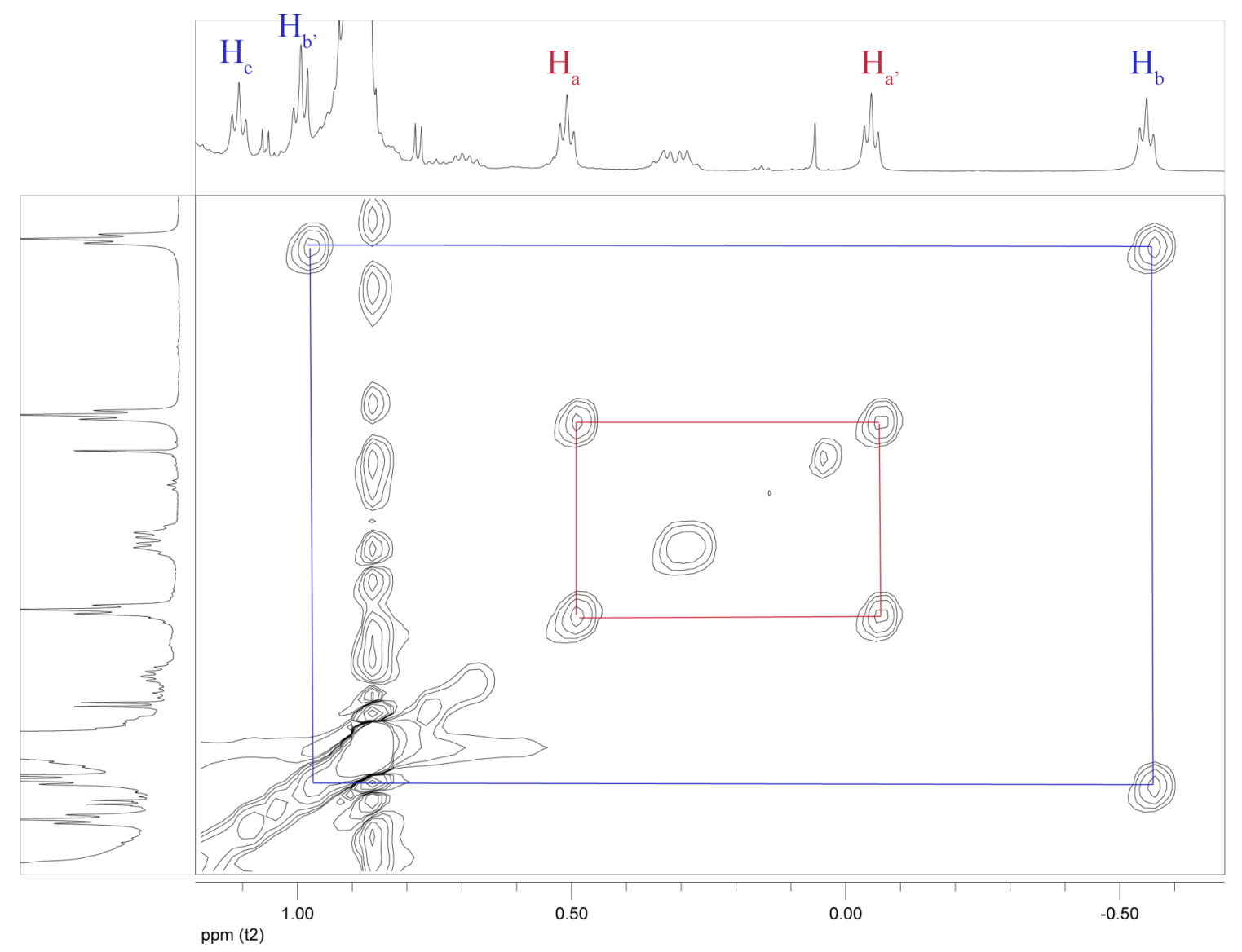

Figure $\boldsymbol{S}$-21. Upfield region of the 2D NOESY spectrum of isobutyl imine cavitand 15i, showing chemical exchange between amide Me peaks. 


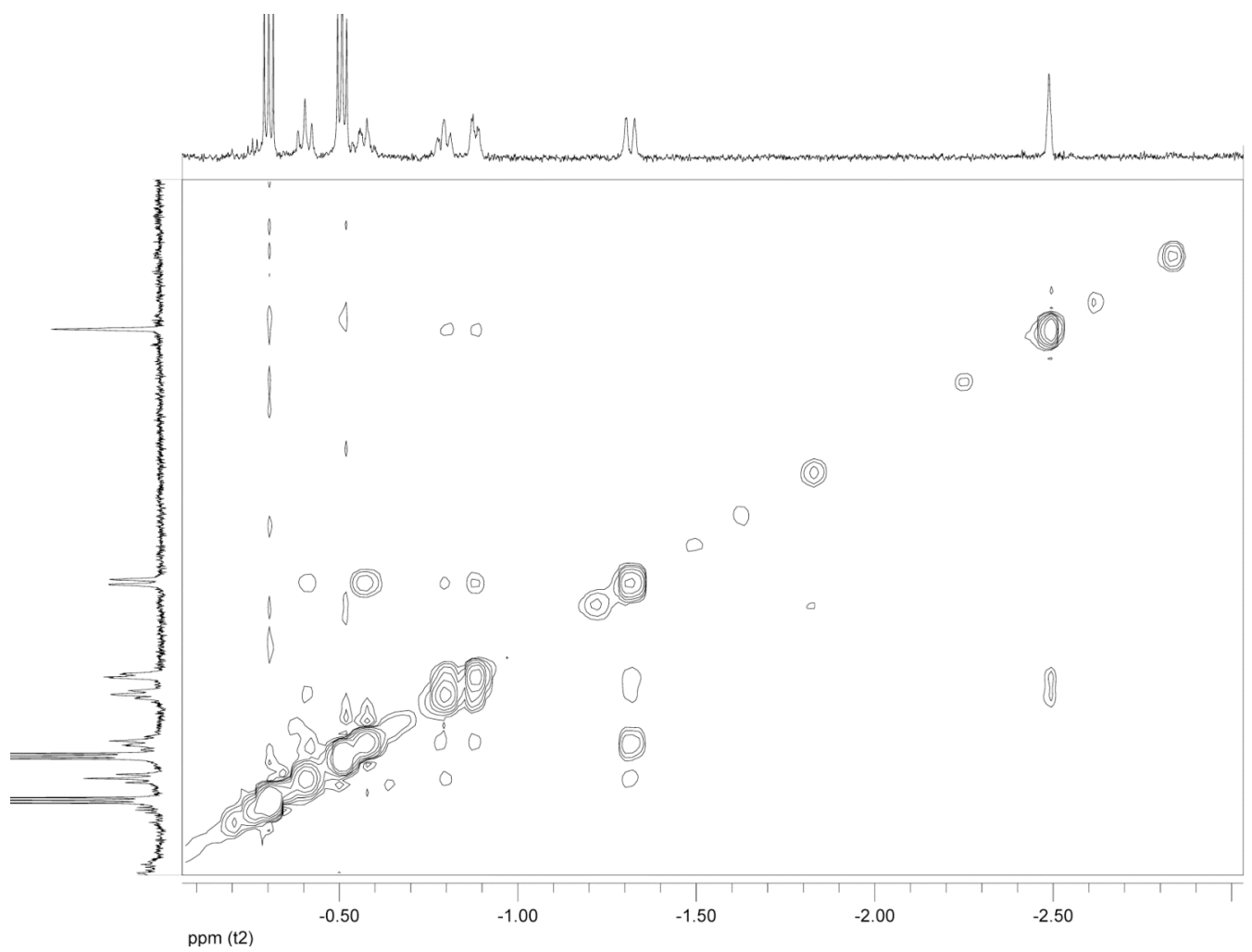

Figure $\boldsymbol{S}$-22. Upfield region of the 2D NOESY spectrum of the hemiaminal 24 h formed by addition of ethanolamine to a $1.5 \mathrm{mM}$ solution of cavitand $\mathbf{3}$ in mesitylene- $d_{12}$. 


\section{Tabulated Rate Data}

\subsection{Short-lived Hemiaminals}

Cavitand $\mathbf{3}+$ isobutylamine

$\begin{array}{cccccc}\begin{array}{c}\text { [cav] }= \\ \text { 1.5 mM }\end{array} & \text { [amine] }=15 \mathrm{mM} \\ \text { time/min } & \text { [complex]/mM } & \text { [hemiaminal]/mM } & \text { [imine]/mM } & \text { [aldehyde]/mM } & \text { Ln[hemiaminal] } \\ 0 & 1.50 & 0.00 & 0.00 & 1.50 & \text { N/A } \\ 2 & 0.45 & 1.05 & 0.00 & 1.50 & 0.05 \\ 5 & 0.16 & 1.20 & 0.14 & 1.36 & 0.18 \\ 9 & 0.13 & 1.15 & 0.23 & 1.27 & 0.14 \\ 17 & 0.08 & 1.04 & 0.38 & 1.13 & 0.04 \\ 24 & 0.07 & 0.90 & 0.53 & 0.97 & -0.11 \\ 32 & 0.06 & 0.70 & 0.74 & 0.76 & -0.35 \\ 47 & 0.04 & 0.50 & 0.96 & 0.54 & -0.69 \\ 77 & 0.02 & 0.23 & 1.25 & 0.25 & -1.46 \\ 107 & 0.01 & 0.10 & 1.39 & 0.11 & -2.32 \\ 137 & 0.00 & 0.00 & 1.50 & 0.00 & -6.50\end{array}$

Cavitand $3+n$-propylamine

\begin{tabular}{|c|c|c|c|c|c|}
\hline \multicolumn{2}{|c|}{$[\mathrm{cav}]=1.5 \mathrm{mM}$} & \multicolumn{2}{|c|}{ [amine] $=15 \mathrm{mM}$} & \multirow[b]{2}{*}{ [aldehyde]/mM } & \multirow[b]{2}{*}{ Ln[hemiaminal] } \\
\hline time/min & [complex]/mM & [hemiaminal]/mM & [imine] $/ \mathrm{mM}$ & & \\
\hline 0 & 1.50 & 0.00 & 0.00 & 1.50 & $\mathrm{~N} / \mathrm{A}$ \\
\hline 2 & 0.11 & 1.39 & 0.00 & 1.50 & 0.33 \\
\hline 5 & 0.00 & 1.38 & 0.12 & 1.38 & 0.32 \\
\hline 15 & 0.00 & 1.31 & 0.19 & 1.31 & 0.27 \\
\hline 25 & 0.00 & 1.13 & 0.37 & 1.13 & 0.13 \\
\hline 45 & 0.00 & 0.94 & 0.56 & 0.94 & -0.06 \\
\hline 68 & 0.00 & 0.68 & 0.82 & 0.68 & -0.39 \\
\hline 85 & 0.00 & 0.47 & 1.03 & 0.47 & -0.75 \\
\hline 105 & 0.00 & 0.31 & 1.19 & 0.31 & -1.17 \\
\hline 240 & 0.00 & 0.23 & 1.27 & 0.23 & -1.47 \\
\hline
\end{tabular}




\section{Cavitand $\mathbf{3}+$ isopropylamine}

$\begin{array}{cccccc}\text { [cav] }=1.5 \mathrm{mM} & \text { [amine] }=15 \mathrm{mM} \\ \text { time/min } & \text { [complex]/mM } & \text { [hemiaminal]/mM } & \text { [imine] } / \mathrm{mM} & \text { [aldehyde] } / \mathrm{mM} & \text { Ln[hemiaminal] } \\ 0 & 1.50 & 0.00 & 0.00 & 1.50 & \mathrm{~N} / \mathrm{A} \\ 3 & 0.00 & 1.50 & 0.00 & 1.50 & 0.41 \\ 10 & 0.00 & 1.36 & 0.14 & 1.36 & 0.31 \\ 17 & 0.00 & 1.30 & 0.20 & 1.30 & 0.27 \\ 35 & 0.00 & 1.22 & 0.28 & 1.22 & 0.20 \\ 60 & 0.00 & 1.09 & 0.41 & 1.09 & 0.08\end{array}$

Cavitand $3+n$-butylamine

\begin{tabular}{|c|c|c|c|c|c|}
\hline \multicolumn{2}{|c|}{$[\mathrm{cav}]=1.5 \mathrm{mM}$} & \multicolumn{2}{|c|}{ [amine] $=15 \mathrm{mM}$} & \multirow[b]{2}{*}{ [aldehyde] $/ \mathrm{mM}$} & \multirow[b]{2}{*}{ Ln[hemiaminal] } \\
\hline time/min & [complex]/mM & [hemiaminal]/mM & [imine]/mM & & \\
\hline 0 & 1.50 & 0.00 & 0.00 & 1.50 & $\mathrm{~N} / \mathrm{A}$ \\
\hline 2 & 0.20 & 1.30 & 0.00 & 1.50 & 0.27 \\
\hline 5 & 0.09 & 1.34 & 0.07 & 1.43 & 0.29 \\
\hline 9 & 0.00 & 1.12 & 0.38 & 1.12 & 0.11 \\
\hline 16 & 0.00 & 0.88 & 0.62 & 0.88 & -0.13 \\
\hline 38 & 0.00 & 0.39 & 1.11 & 0.39 & -0.95 \\
\hline 70 & 0.00 & 0.04 & 1.46 & 0.04 & -3.27 \\
\hline 95 & 0.00 & 0.00 & 1.50 & 0.00 & $\mathrm{~N} / \mathrm{A}$ \\
\hline
\end{tabular}

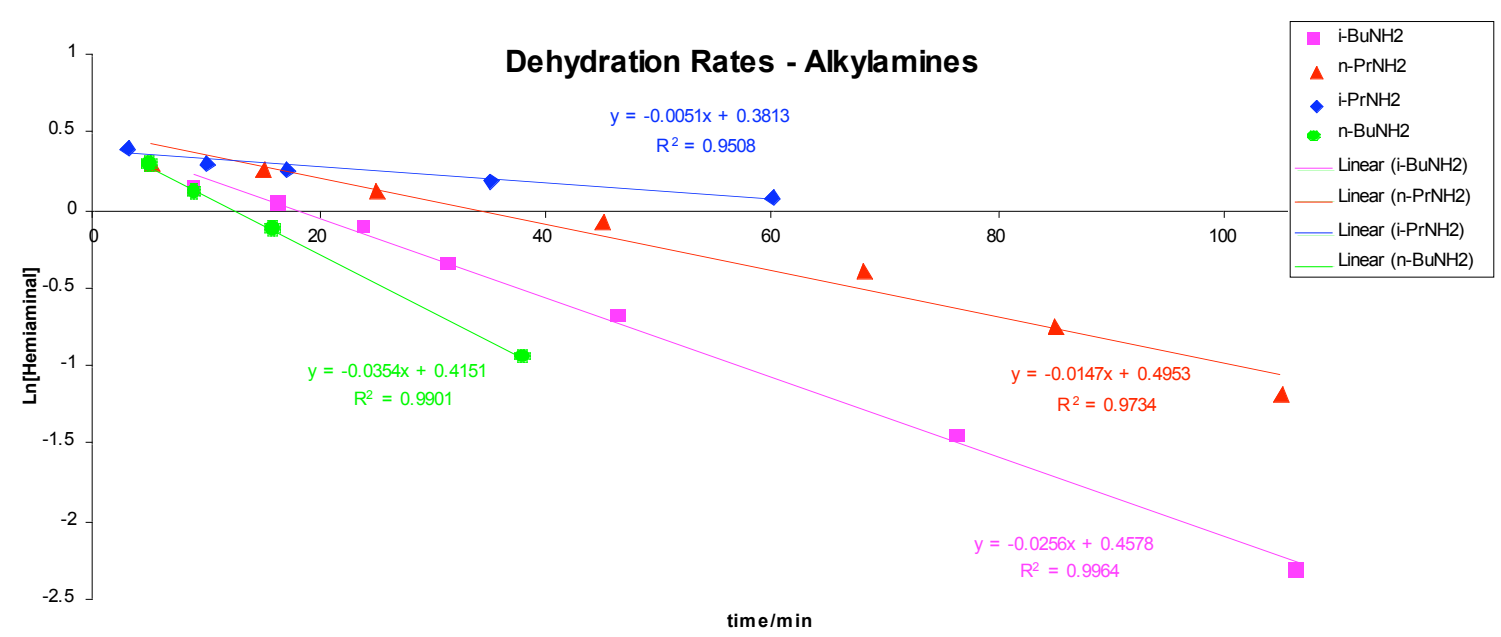

Figure $\boldsymbol{S - 2 3}$. Profiles of the first order dehydration rates of the short-lived hemiaminals from isopropylamine, isobutylamine, $n$-propylamine and $n$-butylamine. 


\subsection{Persistent Hemiaminals}

\section{Cavitand $\mathbf{3}+$ cyclo propylamine}

$\begin{array}{cccc}\text { [cav] = } 1.5 \mathrm{mM} & \text { [amine] }=15 \mathrm{mM} \\ \text { time/min } & \text { [hemiaminal]/mM } & \text { [imine]/mM } & \text { Ln[hemiaminal] } \\ 0 & 1.50 & 0.00 & 0.41 \\ 42 & 1.36 & 0.14 & 0.31 \\ 88 & 1.23 & 0.27 & 0.21 \\ 135 & 1.07 & 0.43 & 0.07 \\ 181 & 0.97 & 0.53 & -0.03 \\ 228 & 0.91 & 0.59 & -0.09 \\ 274 & 0.79 & 0.71 & -0.23 \\ 321 & 0.71 & 0.79 & -0.35 \\ 367 & 0.65 & 0.85 & -0.43 \\ 414 & 0.58 & 0.92 & -0.54 \\ 460 & 0.51 & 0.99 & -0.67 \\ 507 & 0.44 & 1.06 & -0.82 \\ 553 & 0.41 & 1.09 & -0.88 \\ 600 & 0.35 & 1.15 & -1.05 \\ 646 & 0.32 & 1.18 & -1.15 \\ 693 & 0.29 & 1.21 & -1.24 \\ 739 & 0.23 & 1.27 & -1.47\end{array}$

Cavitand $\mathbf{3}+$ cyclo butylamine

$$
\text { [cav] }=1.5 \mathrm{mM} \quad \text { [amine] }=15 \mathrm{mM}
$$

$\begin{array}{cccc}\text { time } / \mathrm{min} & \text { [hemiaminal] } / \mathrm{mM} & \text { [imine] } / \mathrm{mM} & \text { Ln[hemiaminal] } \\ 0 & 1.50 & 0.00 & 0.41 \\ 42 & 1.46 & 0.04 & 0.38 \\ 88 & 1.35 & 0.15 & 0.30 \\ 135 & 1.25 & 0.25 & 0.22 \\ 181 & 1.13 & 0.37 & 0.12 \\ 228 & 1.09 & 0.41 & 0.08 \\ 274 & 1.03 & 0.47 & 0.03 \\ 321 & 0.94 & 0.56 & -0.06 \\ 367 & 0.88 & 0.62 & -0.13 \\ 414 & 0.79 & 0.71 & -0.24 \\ 460 & 0.76 & 0.74 & -0.27 \\ 507 & 0.71 & 0.79 & -0.34 \\ 553 & 0.67 & 0.83 & -0.40 \\ 600 & 0.60 & 0.90 & -0.50 \\ 646 & 0.56 & 0.94 & -0.59 \\ 693 & 0.51 & 0.99 & -0.67 \\ 739 & 0.45 & 1.05 & -0.80 \\ 786 & 0.40 & 1.10 & -0.91 \\ 832 & 0.38 & 1.12 & -0.97 \\ 879 & 0.35 & 1.15 & -1.06 \\ 925 & 0.32 & 1.18 & -1.15\end{array}$




\section{Cavitand 3 + ethylenediamine}

\begin{tabular}{cccc} 
[cav] $=1.5 \mathrm{mM}$ & \multicolumn{3}{c}{ [amine] $=15 \mathrm{mM}$} \\
time/min & [Hemiaminal]/mM & [imine] $/ \mathrm{mM}$ & Ln[hemiaminal] \\
0 & 1.50 & 0.00 & 0.41 \\
15 & 1.50 & 0.00 & 0.41 \\
1165 & 1.32 & 0.18 & 0.27 \\
2630 & 1.07 & 0.43 & 0.07 \\
6910 & 0.61 & 0.89 & -0.49 \\
8330 & 0.56 & 0.94 & -0.58
\end{tabular}

Cavitand 3 + ethanolamine

$\begin{array}{cccc}\text { [cav] }=1.5 \mathrm{mM} & \text { [amine] }=15 \mathrm{mM} \\ \text { time/min } & \text { [Hemiaminal]/mM } & \text { [imine]/mM } & \text { Ln[hemiaminal] } \\ 0 & 1.50 & 0.00 & 0.41 \\ 45 & 1.50 & 0.00 & 0.41 \\ 1155 & 1.08 & 0.42 & 0.08 \\ 2650 & 0.81 & 0.69 & -0.22 \\ 6910 & 0.41 & 1.09 & -0.90 \\ 8265 & 0.27 & 1.23 & -1.31\end{array}$

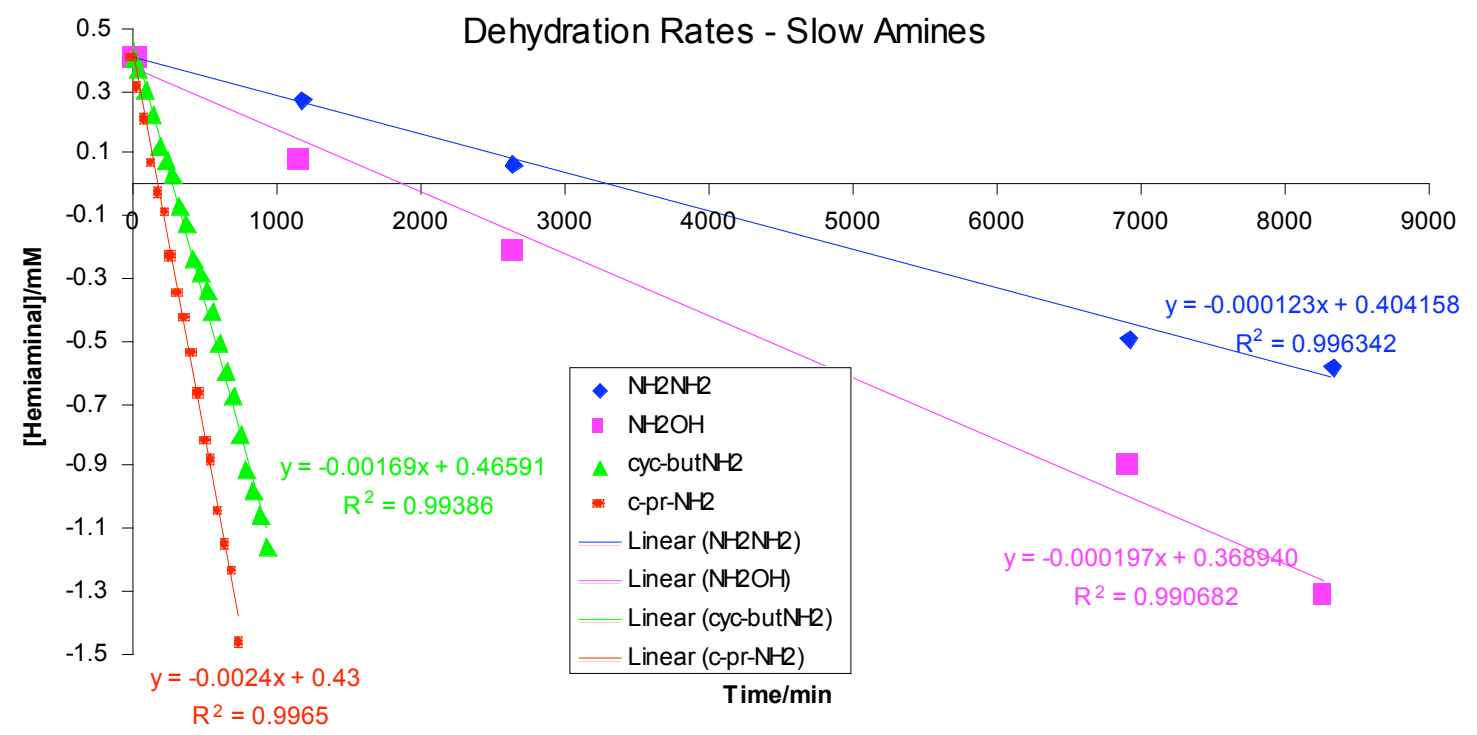

Figure $\boldsymbol{S}$-24. Profiles of the first order dehydration rates of the persistent hemiaminals from ethylenediamine, ethanolamine, cyclopropylamine and cyclobutylamine. 


\subsection{Extroverted Imine Formation Rates}

\section{Cavitand $\mathbf{3}+$ hexylamine in toluene}

\begin{tabular}{|c|c|c|c|c|}
\hline \multicolumn{2}{|c|}{$[\mathrm{cav}]=1.5 \mathrm{mM}$} & \multicolumn{2}{|c|}{ [hexylamine] $=7 \mathrm{mM}$} & \multirow[b]{2}{*}{ linearization } \\
\hline time/min & [aldehyde]/mM & [amine]/mM & [imine] $/ \mathrm{mM}$ & \\
\hline 0 & 1.50 & 7.00 & 0.00 & 0.00 \\
\hline 3 & 1.49 & 6.99 & 0.01 & 0.00 \\
\hline 5 & 1.46 & 6.96 & 0.04 & 0.00 \\
\hline 8 & 1.43 & 6.93 & 0.07 & 0.01 \\
\hline 12 & 1.32 & 6.82 & 0.18 & 0.02 \\
\hline 17 & 1.25 & 6.75 & 0.25 & 0.03 \\
\hline 22 & 1.22 & 6.72 & 0.28 & 0.03 \\
\hline 30 & 1.10 & 6.60 & 0.40 & 0.05 \\
\hline 40 & 0.98 & 6.48 & 0.52 & 0.06 \\
\hline 49 & 0.92 & 6.42 & 0.58 & 0.07 \\
\hline 65 & 0.74 & 6.24 & 0.76 & 0.11 \\
\hline 84 & 0.61 & 6.11 & 0.89 & 0.14 \\
\hline \multicolumn{2}{|c|}{$[\mathrm{cav}]=1.5 \mathrm{mM}$} & \multicolumn{2}{|c|}{ [hexylamine] $=19 \mathrm{mM}$} & \\
\hline time/min & [aldehyde]/mM & [amine]/mM & [imine] $/ \mathrm{mM}$ & linearization \\
\hline 0 & 1.50 & 19.00 & 0.00 & 0.00 \\
\hline 3 & 1.44 & 18.94 & 0.06 & 0.00 \\
\hline 5 & 1.38 & 18.88 & 0.12 & 0.00 \\
\hline 8 & 1.32 & 18.82 & 0.18 & 0.01 \\
\hline 12 & 1.23 & 18.73 & 0.27 & 0.01 \\
\hline 17 & 1.08 & 18.58 & 0.42 & 0.02 \\
\hline 22 & 1.01 & 18.51 & 0.49 & 0.02 \\
\hline 30 & 0.85 & 18.35 & 0.65 & 0.03 \\
\hline 40 & 0.72 & 18.22 & 0.78 & 0.04 \\
\hline 49 & 0.59 & 18.09 & 0.91 & 0.05 \\
\hline 65 & 0.44 & 17.94 & 1.06 & 0.07 \\
\hline 84 & 0.29 & 17.79 & 1.21 & 0.09 \\
\hline \multicolumn{2}{|c|}{$[\mathrm{cav}]=1.5 \mathrm{mM}$} & \multicolumn{2}{|c|}{ [hexylamine] $=32 \mathrm{mM}$} & \\
\hline time/min & [aldehyde]/mM & [amine] $/ \mathrm{mM}$ & [imine] $/ \mathrm{mM}$ & linearization \\
\hline 0 & 1.50 & 32.00 & 0.00 & 0.00 \\
\hline 3 & 1.39 & 31.89 & 0.11 & 0.00 \\
\hline 5 & 1.28 & 31.78 & 0.22 & 0.00 \\
\hline 8 & 1.22 & 31.72 & 0.28 & 0.01 \\
\hline 12 & 1.06 & 31.56 & 0.44 & 0.01 \\
\hline 17 & 0.94 & 31.44 & 0.56 & 0.01 \\
\hline 22 & 0.82 & 31.32 & 0.68 & 0.02 \\
\hline 30 & 0.65 & 31.15 & 0.85 & 0.03 \\
\hline 40 & 0.47 & 30.97 & 1.03 & 0.04 \\
\hline 49 & 0.35 & 30.85 & 1.15 & 0.05 \\
\hline 65 & 0.22 & 30.72 & 1.28 & 0.06 \\
\hline 84 & 0.13 & 30.63 & 1.37 & 0.08 \\
\hline
\end{tabular}




\begin{tabular}{ccccc} 
[cav] $=$ & $\mathbf{1 . 5} \mathrm{mM}$ & \multicolumn{3}{c}{ [hexylamine] $=47 \mathrm{mM}$} \\
time/min & [aldehyde] $/ \mathrm{mM}$ & [amine] $/ \mathrm{mM}$ & [imine] $/ \mathrm{mM}$ & linearization \\
0 & 1.50 & 47.00 & 0.00 & 0.00 \\
3 & 1.35 & 46.85 & 0.15 & 0.00 \\
5 & 1.23 & 46.73 & 0.27 & 0.00 \\
8 & 1.02 & 46.52 & 0.48 & 0.01 \\
12 & 0.77 & 46.27 & 0.73 & 0.01 \\
17 & 0.66 & 46.16 & 0.84 & 0.02 \\
22 & 0.46 & 45.96 & 1.04 & 0.03 \\
30 & 0.28 & 45.78 & 1.22 & 0.04
\end{tabular}

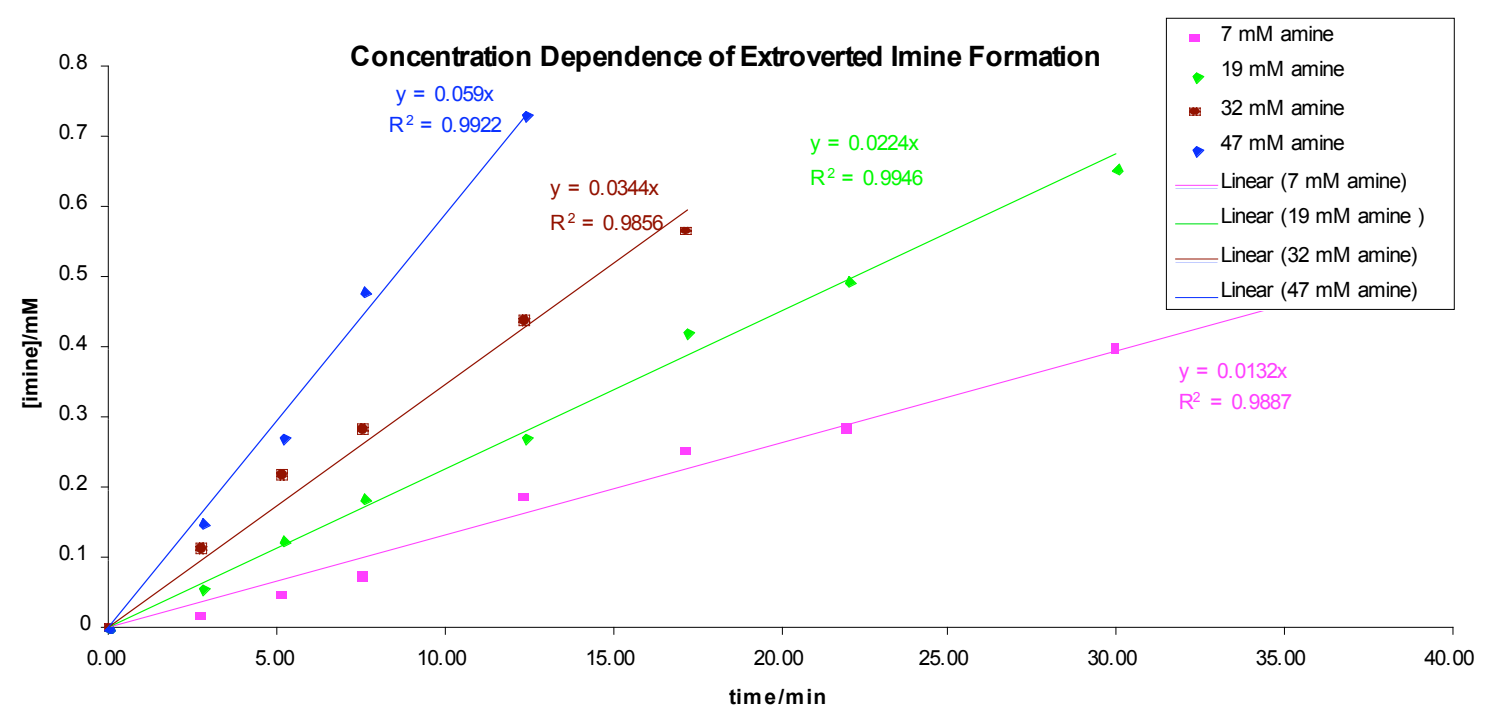

Figure $S$-25. Profiles of the second order rates of imine formation with $n$-hexylamine at various concentrations of amine $\left(300 \mathrm{~K},[3]=1.5 \mathrm{mM}\right.$, toluene- $\left.d_{8}\right)$. 


\subsection{Competition Experiments}

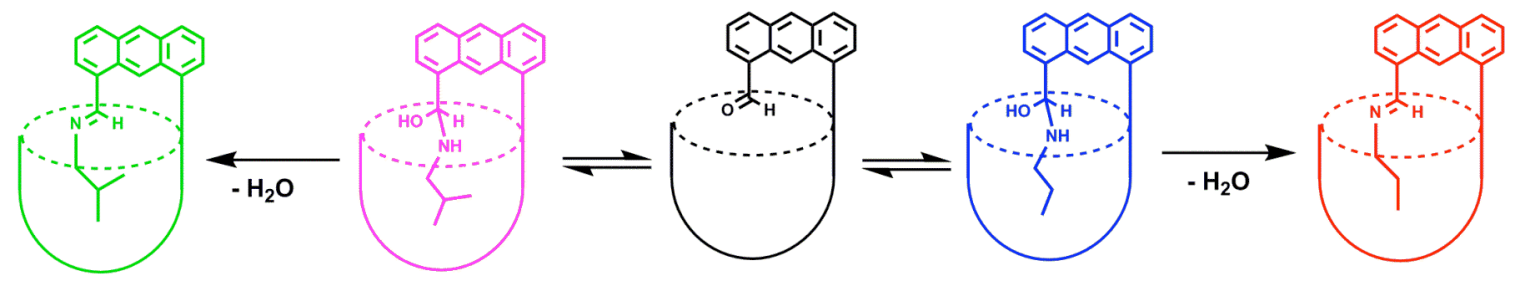

\section{'BuNH ${ }_{2}$ vs ${ }^{\mathrm{n}} \mathrm{PrNH}_{2}$}

\begin{tabular}{|c|c|c|c|c|}
\hline time/min & {$[\mathrm{HA} \mathrm{Bu}] / \mathrm{mM}$} & {$[\mathrm{HA}$ Pr] $/ \mathrm{mM}$} & [imine $\mathrm{Bu}] / \mathrm{mM}$ & [imine $\mathrm{Pr}] / \mathrm{mM}$ \\
\hline 0 & 0.00 & 0.00 & 0.00 & 0.00 \\
\hline 2 & 0.53 & 0.67 & 0.00 & 0.00 \\
\hline 5 & 0.44 & 0.42 & 0.23 & 0.04 \\
\hline 15 & 0.22 & 0.26 & 0.61 & 0.08 \\
\hline 28 & 0.13 & 0.16 & 0.91 & 0.10 \\
\hline 40 & 0.10 & 0.09 & 1.09 & 0.13 \\
\hline 58 & 0.07 & 0.06 & 1.21 & 0.12 \\
\hline 80 & 0.05 & 0.03 & 1.27 & 0.15 \\
\hline 105 & 0.00 & 0.00 & 1.35 & 0.15 \\
\hline
\end{tabular}

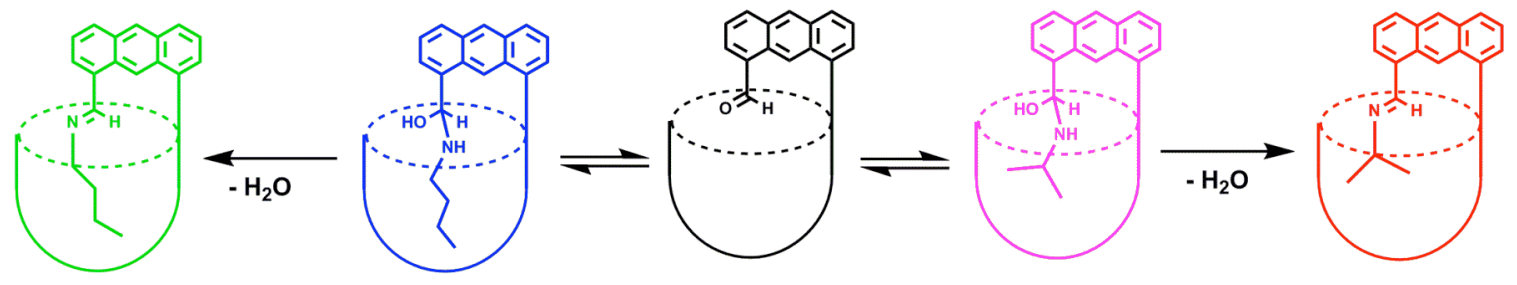

\section{'PrNH ${ }_{2}$ vs ${ }^{\mathrm{n}} \mathrm{BuNH}_{2}$}

\begin{tabular}{|c|c|c|c|c|}
\hline time/min & {$[\mathrm{HA} \mathrm{Bu}] / \mathrm{mM}$} & {$[\mathrm{HA} \mathrm{Pr}] / \mathrm{mM}$} & [imine $\mathrm{Bu}] / \mathrm{mM}$ & [imine $\mathrm{Pr}] / \mathrm{mM}$ \\
\hline 0 & 0.00 & 0.00 & 0.00 & 0.00 \\
\hline 3 & 0.51 & 0.75 & 0.00 & 0.00 \\
\hline 5 & 0.28 & 0.81 & 0.18 & 0.00 \\
\hline 8 & 0.23 & 0.74 & 0.29 & 0.00 \\
\hline 12 & 0.18 & 0.63 & 0.42 & 0.00 \\
\hline 17 & 0.12 & 0.60 & 0.43 & 0.10 \\
\hline 22 & 0.10 & 0.47 & 0.57 & 0.17 \\
\hline 30 & 0.08 & 0.39 & 0.69 & 0.17 \\
\hline 40 & 0.06 & 0.28 & 0.87 & 0.18 \\
\hline 49 & 0.05 & 0.23 & 0.92 & 0.21 \\
\hline 65 & 0.03 & 0.16 & 1.04 & 0.23 \\
\hline
\end{tabular}




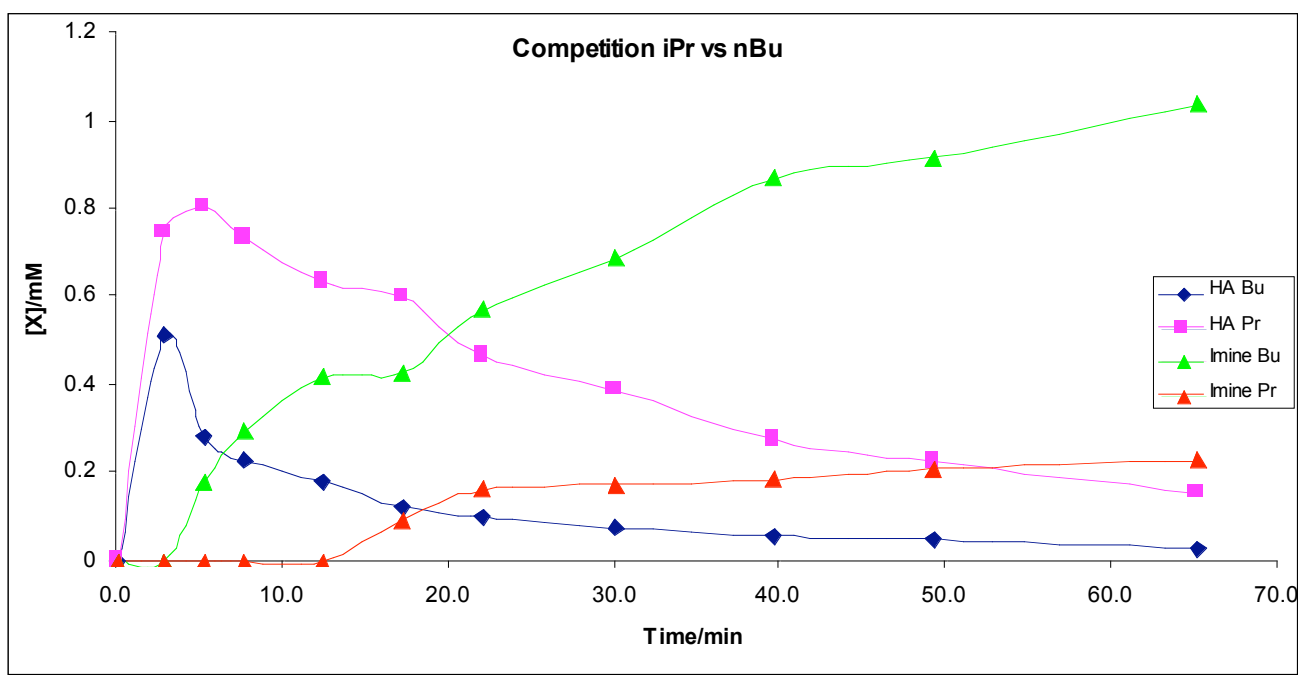

Figure $S$-26. Rate profile of the competing imine formation between equimolar isopropylamine and $n$ butylamine.

\subsection{Transimination Rates}

\section{Extroverted Imine $25+{ }^{\mathrm{i}} \mathrm{BuNH}_{2}$}

\begin{tabular}{cccc} 
[imine] $=1.5 \mathrm{mM}$ & \multicolumn{3}{c}{$[\mathrm{iBuNH} 2]=20 \mathrm{mM}$} \\
time/min & [ext. imine]/mM & [prod] $/ \mathrm{mM}$ & Ln[SM] \\
0 & 1.50 & 0.00 & 0.41 \\
5 & 1.44 & 0.06 & 0.36 \\
15 & 1.40 & 0.10 & 0.33 \\
35 & 1.34 & 0.16 & 0.29 \\
60 & 1.26 & 0.24 & 0.23 \\
88 & 1.15 & 0.35 & 0.14
\end{tabular}

$$
\mathrm{k}=0.14 \times 10^{-3} \mathrm{~min}^{-1}
$$




\begin{tabular}{|c|c|c|c|}
\hline \multicolumn{2}{|c|}{ [imine $]=1.5 \mathrm{mM}$} & \multicolumn{2}{|c|}{$[\mathrm{iBuNH} 2]=20 \mathrm{mM}$} \\
\hline time/min & [start. imine] $/ \mathrm{mM}$ & [prod] $/ \mathrm{mM}$ & $\operatorname{Ln}[\mathrm{SM}]$ \\
\hline 0 & 1.50 & 0.00 & 0.41 \\
\hline 105 & 1.48 & 0.02 & 0.39 \\
\hline 4265 & 1.38 & 0.12 & 0.32 \\
\hline 8475 & 1.16 & 0.34 & 0.15 \\
\hline 9840 & 1.09 & 0.41 & 0.09 \\
\hline 14150 & 0.95 & 0.55 & -0.05 \\
\hline
\end{tabular}

